Network Working Group

Request for Comments: 3295

Category: Standards Track
H. Sjostrand

ipUnplugged

J. Buerkle

Nortel Networks

B. Srinivasan

Cplane

June 2002

\title{
Definitions of Managed Objects for \\ the General Switch Management Protocol (GSMP)
}

Status of this Memo

This document specifies an Internet standards track protocol for the Internet community, and requests discussion and suggestions for improvements. Please refer to the current edition of the "Internet Official Protocol standards" (STD 1) for the standardization state and status of this protocol. Distribution of this memo is unlimited.

Copyright Notice

Copyright (C) The Internet Society (2002). All Rights Reserved.

Abstract

This memo defines a portion of the Management Information Base (MIB)

for the use with the network management protocols in the Internet community. In particular, it describes managed objects for the General Switch Management Protocol (GSMP).

Table of Contents

1. Introduction.......................... 2

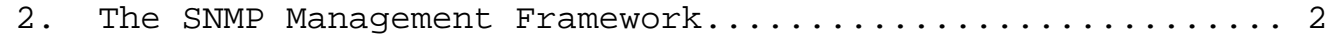

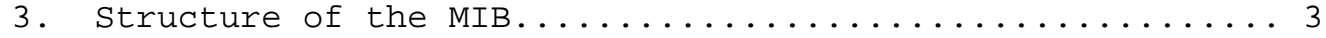

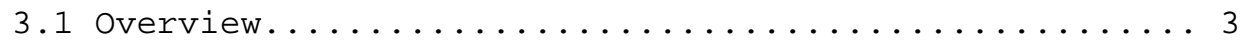

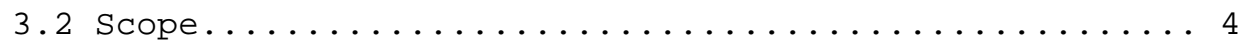

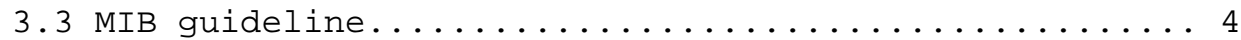

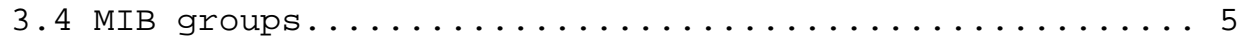

3.4.1 GSMP Switch Controller group.............. 5

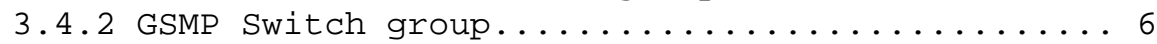

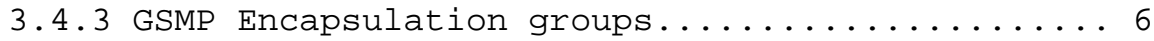

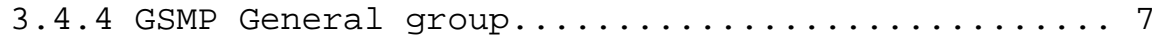

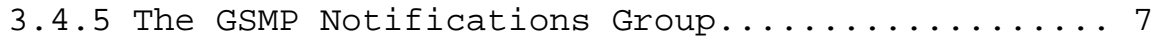

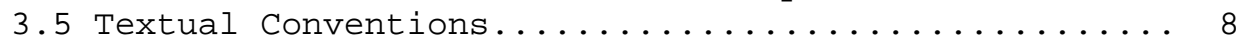

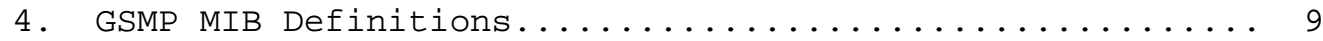

5. Acknowledgments........................ 42 
6. References........................... 42

7. Intellectual Property Rights.................. 44

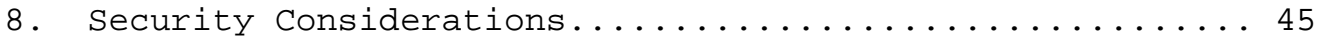

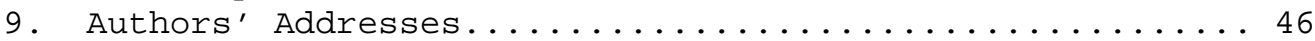

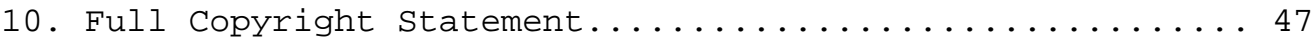

1. Introduction

This memo defines a portion of the Management Information Base (MIB) for use with network management protocols in the Internet community. In particular, it describes managed objects for the General Switch Management Protocol (GSMP).

The key words "MUST", "MUST NOT", "REQUIRED", "SHALL", "SHALL NOT", "SHOULD", "SHOULD NOT", "RECOMMENDED", "MAY", and "OPTIONAL" in this document are to be interpreted as described in RFC 2119 [RFC2119].

2. The SNMP Management Framework

The SNMP Management Framework presently consists of five major components:

* An overall architecture, described in RFC 2571 [RFC2571].

* Mechanisms for describing and naming objects and events for the purpose of management. The first version of this structure of Management Information (SMI) is called SMIV1 and is described in $\mathrm{STD} 16, \mathrm{RFC} 1155$ [RFC1155], STD 16, RFC 1212 [RFC1212], and RFC 1215 [RFC1215]. The second version, called SMIV2, is described in STD 58, RFC 2578 [RFC2578], RFC 2579 [RFC2579], and RFC $2580[\mathrm{RFC} 2580]$.

* Message protocols for transferring management information. The first version of the SNMP message protocol is called SNMPv1 and is described in STD 15, RFC 1157 [RFC1157]. A second version of the SNMP message protocol, which is not an Internet standards track protocol, is called SNMPV2C and is described in RFC 1901 [RFC1901] and RFC 1906 [RFC1906]. The third version of the message protocol is called SNMPv3 and is described in RFC 1906 [RFC1906], RFC 2572 [RFC2572], and RFC 2574 [RFC2574].

* Protocol operations for accessing management information. The first set of protocol operations and associated PDU formats are described in STD 15, RFC 1157 [RFC1157]. A second set of operations and associated PDU formats are described in 1905 [RFC1905]. 
* A set of fundamental applications described in RFC 2573 [RFC2573], and the view-based access control mechanism is described in RFC 2575 [RFC2575].

A more detailed introduction to the current SNMP Management Framework can be found in RFC 2570 [RFC2570].

Managed objects are accessed via a virtual information store, termed the Management Information Base or MIB. Objects in the MIB are defined using the mechanisms defined in the SMI.

This memo specifies a MIB module that is compliant to the SMIV2. A MIB conforming to the SMIv1 can be produced through the appropriate translations. The resulting translated MIB must be semantically equivalent, except where objects or events are omitted because no translation is possible (use of Counter64). Some machine readable information in SMIv2 will be converted into textual descriptions in SMIv1 during the translation process. However, this loss of machine readable information is not considered to change the semantics of the MIB.

3. Structure of the MIB

This memo defines a portion of the Management Information Base (MIB) for the use with network management protocols in the Internet community. In particular, it describes managed objects for the General Switch Management Protocol (GSMP), as defined in [RFC3292].

\subsection{Overview}

The General Switch Management Protocol (GSMP) is a general purpose protocol to control a label switch. GSMP allows a controller to establish and release connections across the switch, to manage switch ports and to request configuration information or statistics. It also allows the switch to inform the controller of asynchronous events such as a link going down.

The GSMP protocol is asymmetric, the controller being the master and the switch being the slave. Multiple switches may be controlled by a single controller using multiple instantiations of the protocol over separate control connections. Also a switch may be controlled by more than one controller by using the technique of partitioning.

Each instance of a (switch controller, switch partition) adjacency is a session between one switch controller entity and one switch entity. The MIB provides objects to configure/setup these entities to form the GSMP sessions. It also provide objects to monitor these GSMP sessions. 


\subsection{Scope}

The GSMP mib is a protocol mib. It contains objects to configure, monitor, and maintain the GSMP protocol entity. It does not provide any information learned via the protocol, such as "all ports config" information.

The relationships between virtual entities, such as Virtual Switch Entities, and "physical" entities, such as Switch Entities, falls outside of the management of GSMP. This also applies for the management of switch partitions. So this is excluded from the GSMP mib.

It is possible to configure which, and how many Switch Controllers are controlling one Switch since every potential session with the switch has to be represented with an Switch entity. It is, however, not possible to define that one Switch Controller shouldn't allow other Switch controllers to control the same switch or partition on the switch. It is assumed that there are mechanisms that synchronise controllers and the configuration of them. This is outside the scope of this mib.

\subsection{MIB guideline}

Two tables are used to configure potential GSMP sessions depending if you are acting as a GSMP switch controller or a GSMP switch. Each row in these tables initiates a GSMP session.

The entity ID is a 48-bit name that is unique within the operational context of the device. A 48-bit IEEE 802 MAC address, if available, MAY be used for the entity ID. If the Ethernet encapsulation is used, the entity ID MUST be the IEEE 802 MAC address of the interface on which the GSMP session is to be setup.

First, the encapsulation of the potential GSMP session shall be defined. If ATM is used, a row in the gsmpAtmEncapTable has to be created with the index set to the entity ID. The specified resources should be allocated to GSMP. If TCP/IP is used, a row in the gsmpTcpIpEncapTable has to be created with the index set to the entity ID. The specified port shall be allocated to GSMP. No special action is needed if ethernet encapsulation is used.

Then the entity information shall be defined. To create a Switch Entity, an entry in the gsmpSwitchTable is created with the index set to the entity ID. To create a Switch Controller Entity, an entry in the gsmpControllertable is created with the index set to the entity ID. 
When the row status of the GsmpControllerEntry or GsmpSwitchEntry is set to active (e.g., in the case with ATM or TCP/IP there are active rows with a corresponding entity ID), the adjacency protocol of GSMP is started.

Another table, the gsmpsessiontable, shows the actual sessions that are established or are in the process of being established. Each row represents a specific session between an Entity and a peer. This table carries information about the peer, the session, and parameters that were negotiated by the adjacency procedures. The gsmpsessionTable also contains statistical information regarding the session.

This creation order SHOULD be used by all GSMP managers. This is to avoid clash situations in multiple SNMP manager scenarios where different managers may create competing entries in the different tables.

Entities may very well be configured by other means than SNMP, e.g., the cli command. Such configured entities SHOULD be represented as entries in the tables of this mib and SHOULD be possible to query, and MAY be possible to alter with SNMP.

\section{4 MIB groups}

\subsubsection{GSMP Switch Controller group}

The controller group is used to configure a potential GSMP session on a Switch Controller. A row in the gsmpControllertable is created for each such session. If ATM or TCP/IP encapsulation is used, a corresponding row has to be created in these tables before the session adjacency protocol is initiated.

If ATM or TCP/IP is used, encapsulation data is defined in the corresponding encapsulation tables. If ethernet is used, the MAC address of the interface defined for the session is set by the controller ID object.

The adjacency parameters are defined; such as

- Max supported GSMP version.

- Time between the periodic adjacency messages.

- Controller local port number and instance number.

- Whether partitions are being used and the partition ID for the specific partitions this controller is concerned with if partitions are used.

- The resynchronisation strategy for the session is specified. 
The notification mapping is set to specify for with events the corresponding SNMP notifications are sent.

\subsubsection{GSMP Switch group}

The switch group is used to configure a potential GSMP session on a Switch. A row in the gsmpSwitchTable is created for each such session. If ATM or TCP/IP encapsulation is used, a corresponding row has to be created in these tables before the session adjacency protocol is initiated.

If ATM or TCP/IP is used, encapsulation data is defined in the corresponding encapsulation tables. If ethernet is used the MAC address of the interface defined for the session is set by the Switch ID object.

The adjacency parameters are defined; such as

- Max supported GSMP version

- Time between the periodic adjacency messages

- Switch Name, local port number, and instance number.

- Whether partitions are being used and the partition ID for this specific partition if partitions are used.

- The switch type could be set.

- The suggested maximum window size for unacknowledged request messages.

Also, a notification mapping is set to specify for with events the corresponding SNMP notifications are sent.

\subsubsection{GSMP Encapsulation groups}

The ATM Encapsulation Table and the TCP/IP Encapsulation Table provides a way to configure information that are encapsulation specific. The encapsulation data is further specified in [RFC3293].

If ATM encapsulation is used, the interface and the virtual channel are specified.

If TCP/IP is used, the IP address and the port number are specified.

No special config data needed if Ethernet encapsulation is used.

This mib MAY be extended with new, standard or proprietary, GSMP encapsulation types. If a new encapsulation type needs to be added, it SHOULD be done in the form of a new table with the entity ID as an index. A row in that encapsulation table sHould be created before any row in a GSMP entity table is created that is using this new GSMP encapsulation. 


\subsubsection{GSMP General group}

The GSMP session table provides a way to monitor and maintain GSMP sessions.

The session is defined by a Switch Controller Entity and Switch Entity pair.

\subsubsection{The GSMP Notifications Group}

The GSMP Notification Group defines notifications for GSMP entities. These notifications provide a mechanism for a GSMP device to inform the management station of status changes. Also a notification is defined for each type of GSMP events.

The group of notifications consists of the following notifications:

- gsmpSessionDown

This notification is generated when a session is terminating and also reports the final accounting statistics of the session.

- gsmpsessionUp

This notification is generated when a new session is established.

- gsmpSendFailureInd

This notification is generated when a message with a failure indication was sent. This means that this notification identifies a change to the gsmpSessionStatFailureInds object in a row of the gsmpSessionTable.

- gsmpReceivedFailureInd

This notification is generated when a message with a failure indication received. This means that this notification identifies a change to the gsmpsessionstatReceivedFailures object in a row of the gsmpSessionTable.

- gsmpPortUpEvent

This notification is generated when a Port Up Event is either received or sent. 
- gsmpPortDownEvent

This notification is generated when a Port Down Event is either received or sent.

- gsmpInvalidLabelEvent

This notification is generated when an Invalid Label Event is either received or sent.

- gsmpNewPortEvent

This notification is generated when New Port Event either is received or sent.

- gsmpDeadPortEvent

This notification is generated when a Dead Port Event is either received or sent.

- gsmpAdjacencyUpdateEvent

This notification is generated when an Adjacency Update Event is either received or sent.

To disable or enable the sending of each notification, the bits in the bitmap are set to 0 or 1 in the Notification mapping objects in the Controller Entitiy or Switch Entity tables.

The GSMP notification map capability should not be seen as a duplication of the filter mechanism in the snmp notification originator application [RFC2573], but as a compliment, to configure the relation between GSMP events and the SNMP notifications already in the GSMP agent. SNMP notifications and GSMP events operate sometimes on a different timescale, and it may in some applications be devastating for a SNMP application to receive events for each GSMP events. E.g. the invalid label event in a ATM switch scenario may cause mass SNMP notification flooding if mapped to a SNMP notification.

\subsection{Textual Conventions}

The datatypes GsmpNameType, GsmpLabelType, GsmpVersion, GsmpPartitionType, and GsmpPartitionIdType are used as textual conventions in this document. These textual conventions are used for the convenience of humans reading the MIB. Objects defined using these conventions are always encoded by means of the rules that define their primitive type. However, the textual conventions have 
special semantics associated with them. Hence, no changes to the SMI or the SNMP are necessary to accommodate these textual conventions which are adopted merely for the convenience of readers.

4. GSMP MIB Definitions

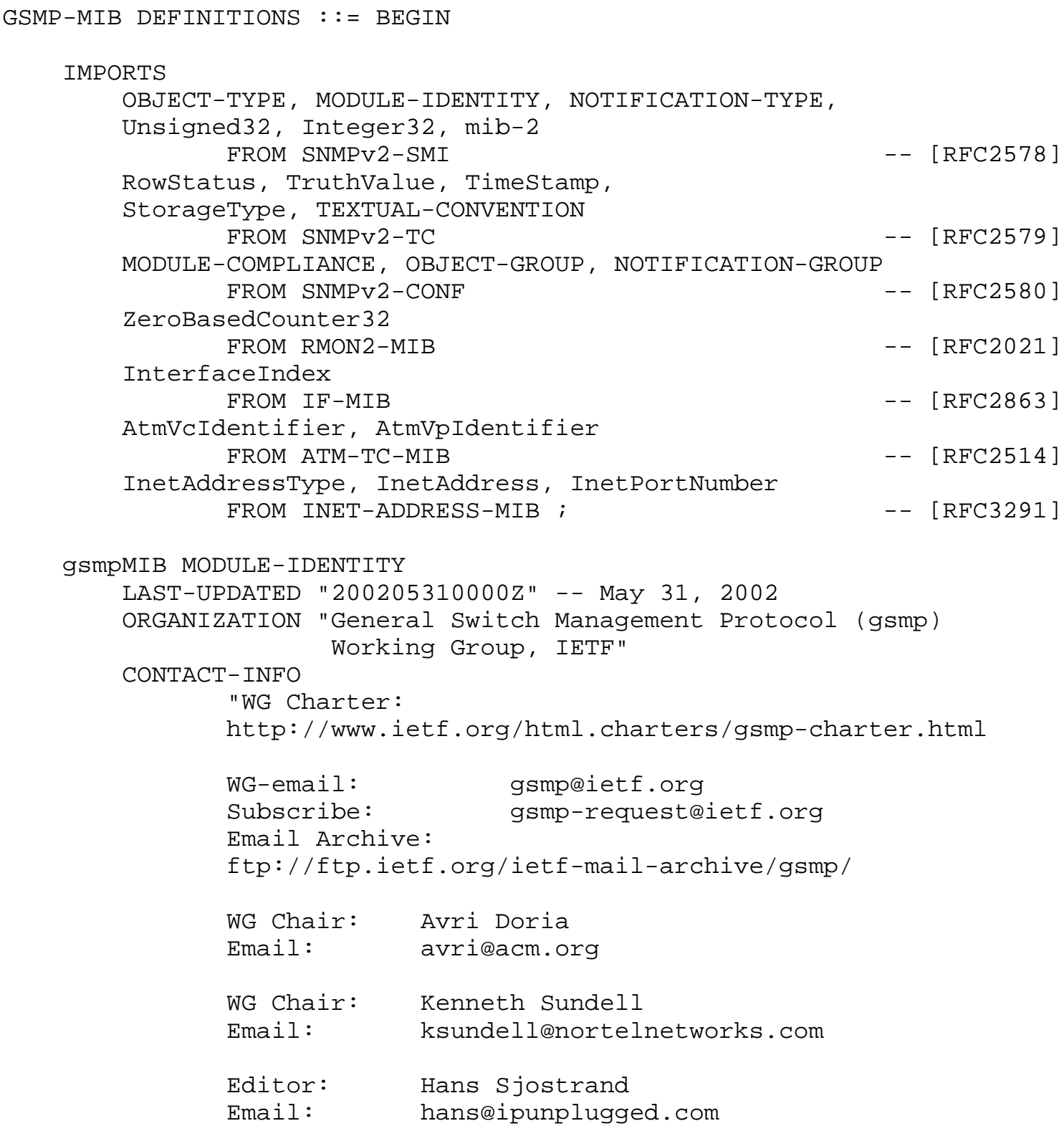

Sjostrand, et. al. Standards Track [Page 9] 


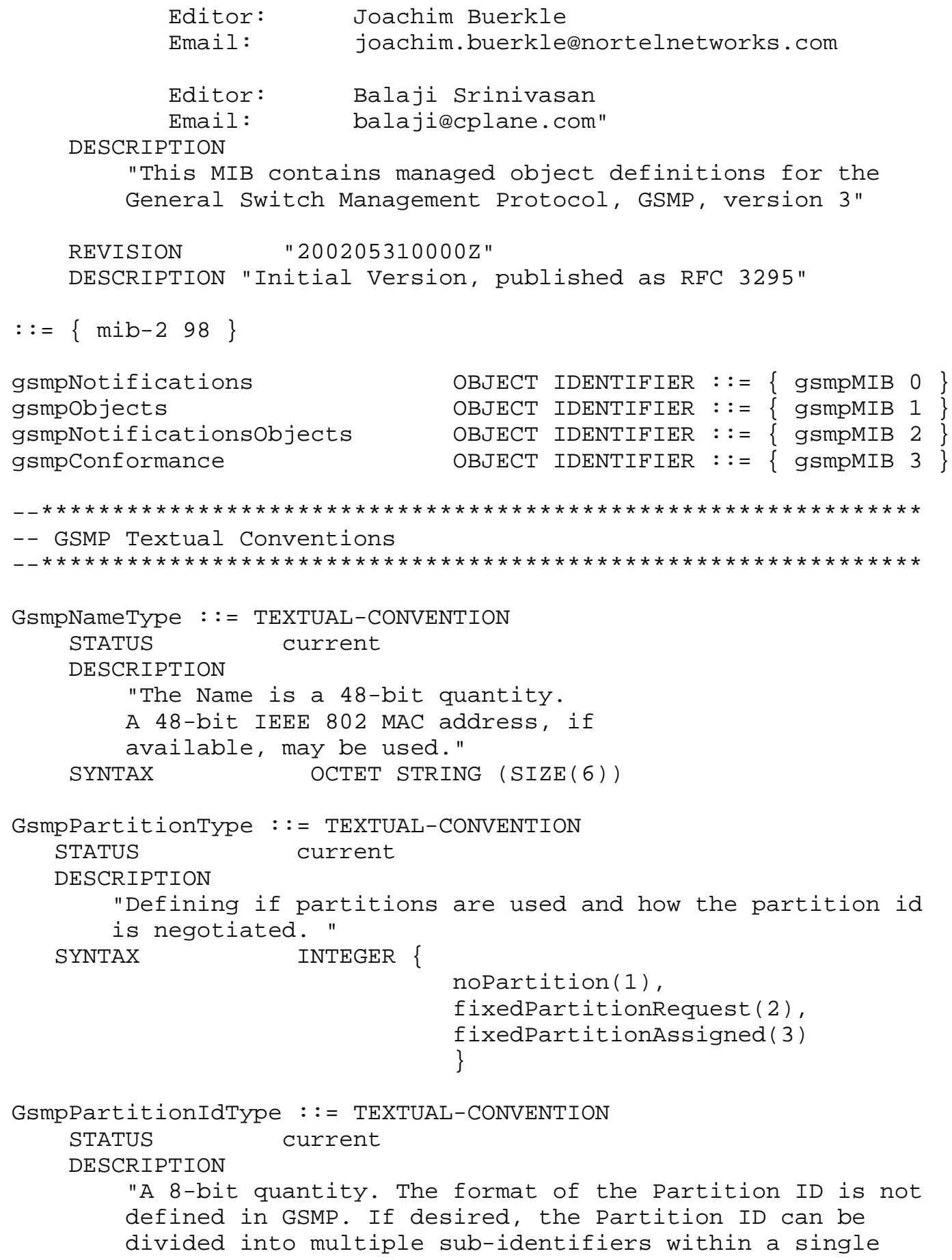


partition. For example: the Partition ID could be subdivided into a 6-bit partition number and a 2-bit sub-identifier which would allow a switch to support 64 partitions with 4 available IDs per partition." SYNTAX OCTET STRING (SIZE(1))

GsmpVersion : := TEXTUAL-CONVENTION

STATUS current

DESCRIPTION

"The version numbers defined for the GSMP protocol.

The version numbers used are defined in the specifications of the respective protocol, 1 - GSMPV1.1 [RFC1987]

2 - GSMPV2.0 [RFC2397]

3 - GSMPv3 [RFC3292]

Other numbers may be defined for other versions

SYNTAX $\quad$ Unsigned 32

GsmpLabelType : := TEXTUAL-CONVENTION

STATUS current

DESCRIPTION

"The label is structured as a TLV, a tuple, consisting of a Type, a Length, and a Value. The structure is defined in [RFC 3292]. The label TLV is encoded as a 2 octet type field, followed by a 2 octet Length field, followed by a variable length Value field.

Additionally, a label field can be composed of many stacked labels that together constitute the label." SYNTAX OCTET STRING

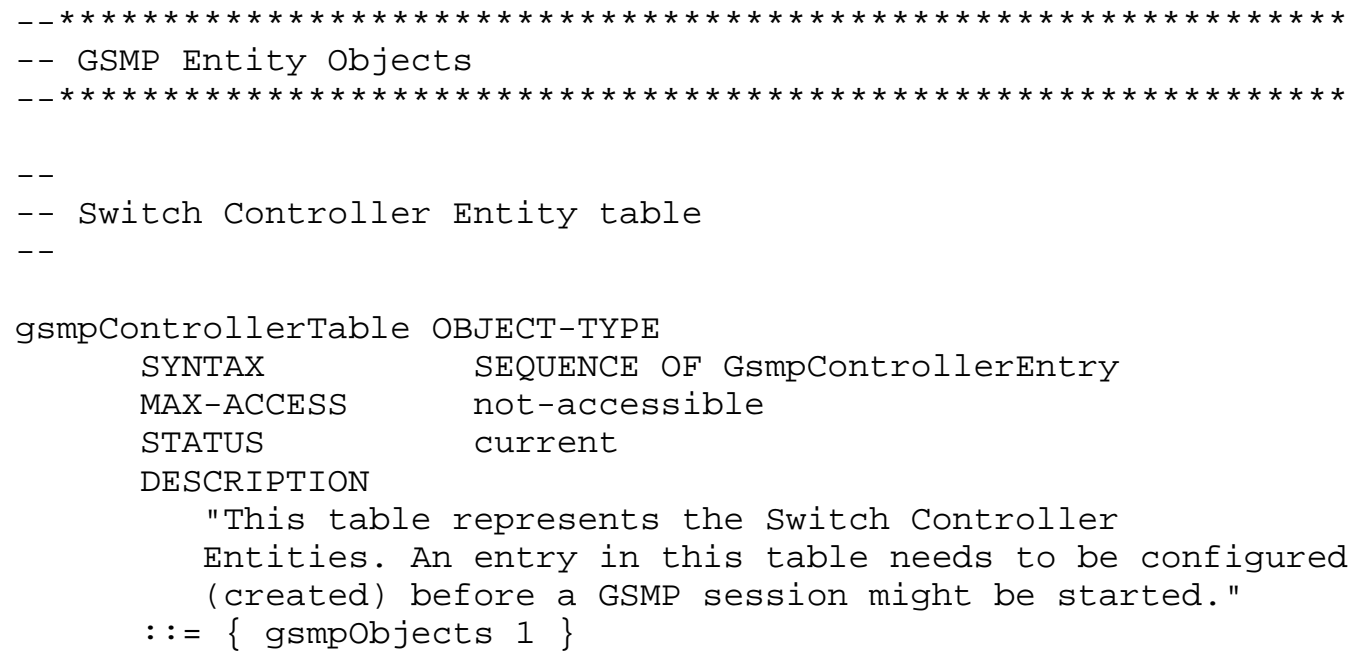




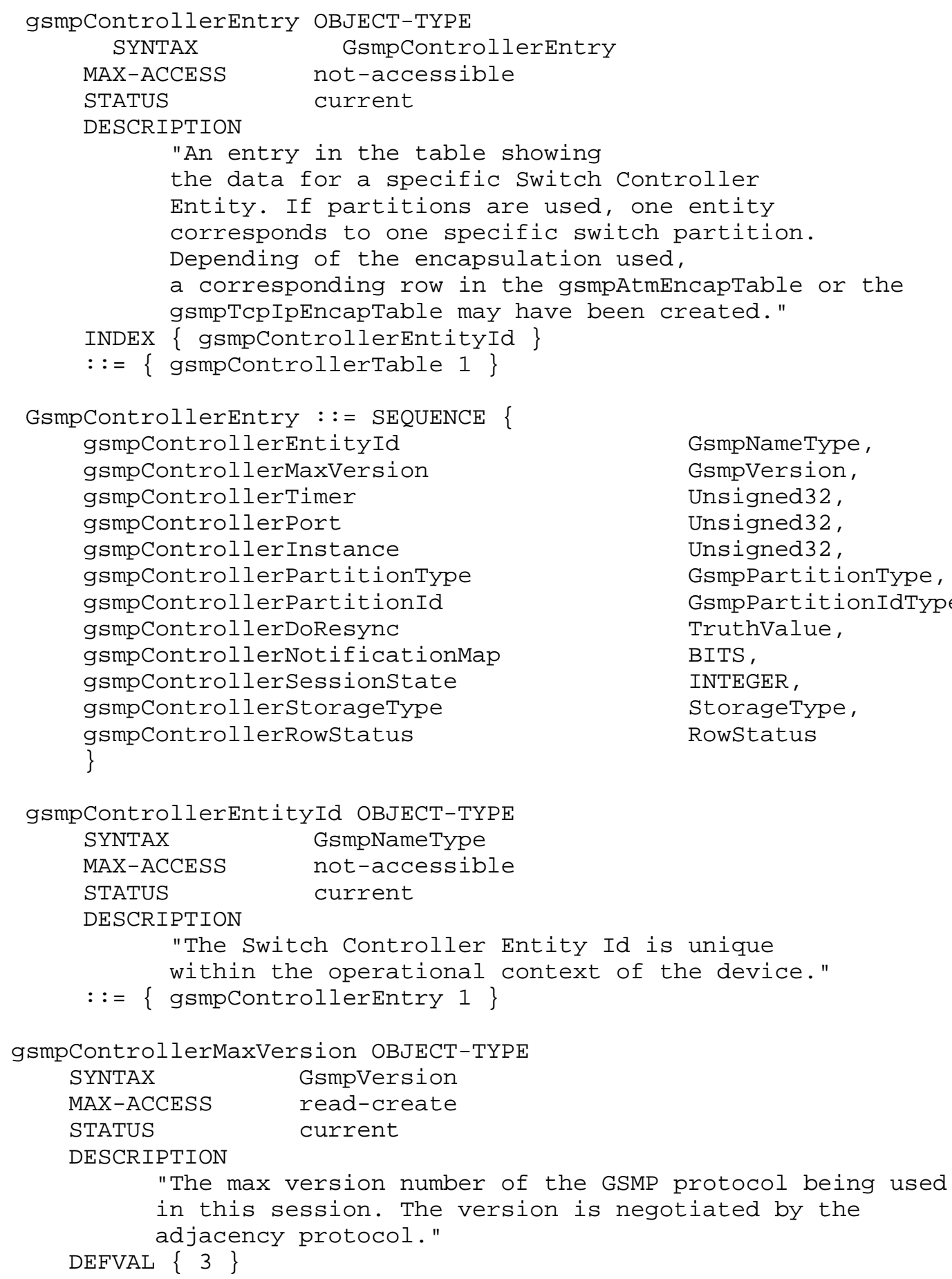


$::=\{$ gsmpControllerEntry 2$\}$

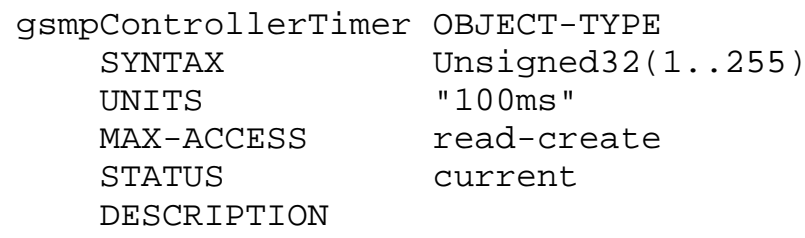




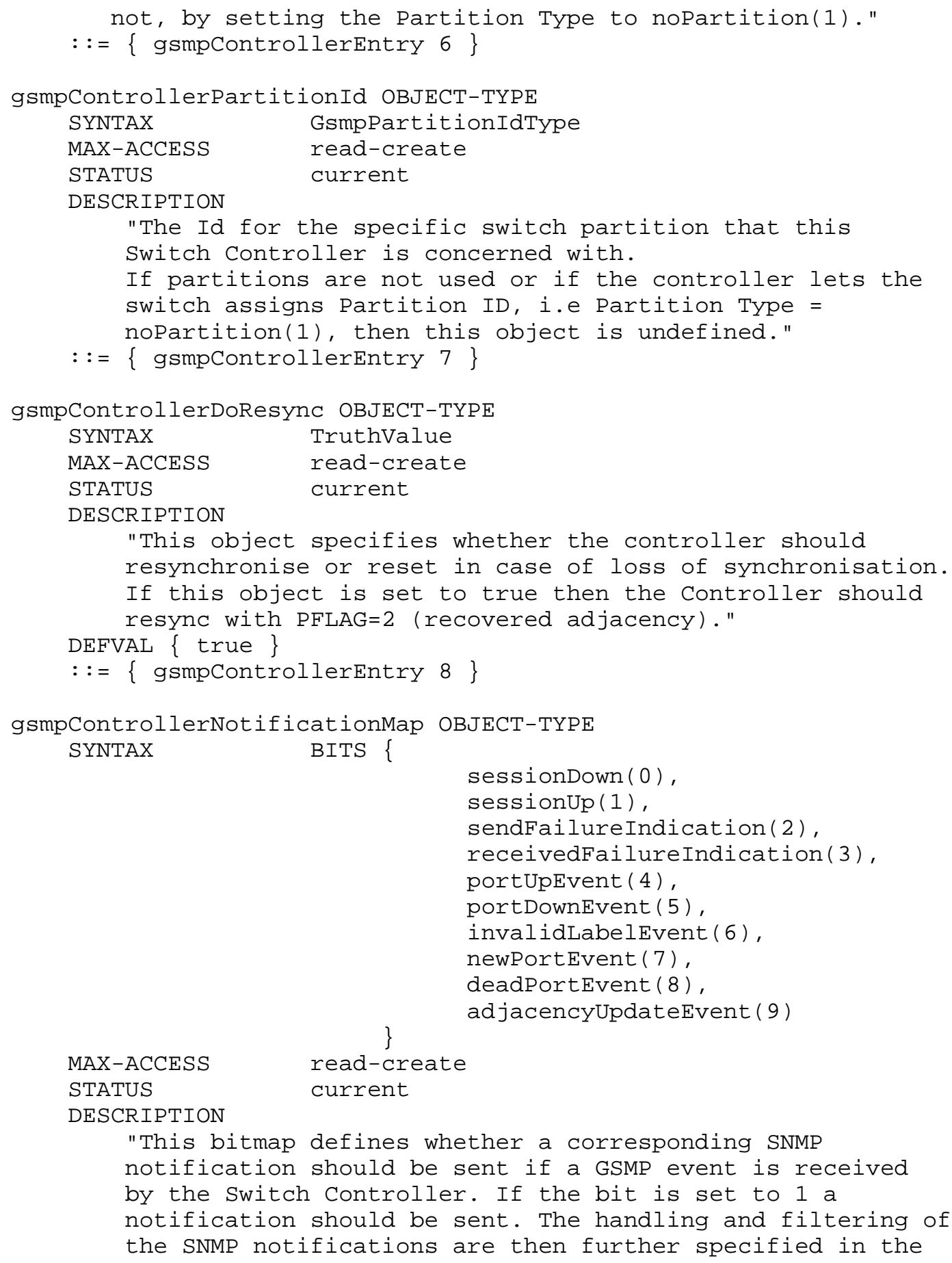




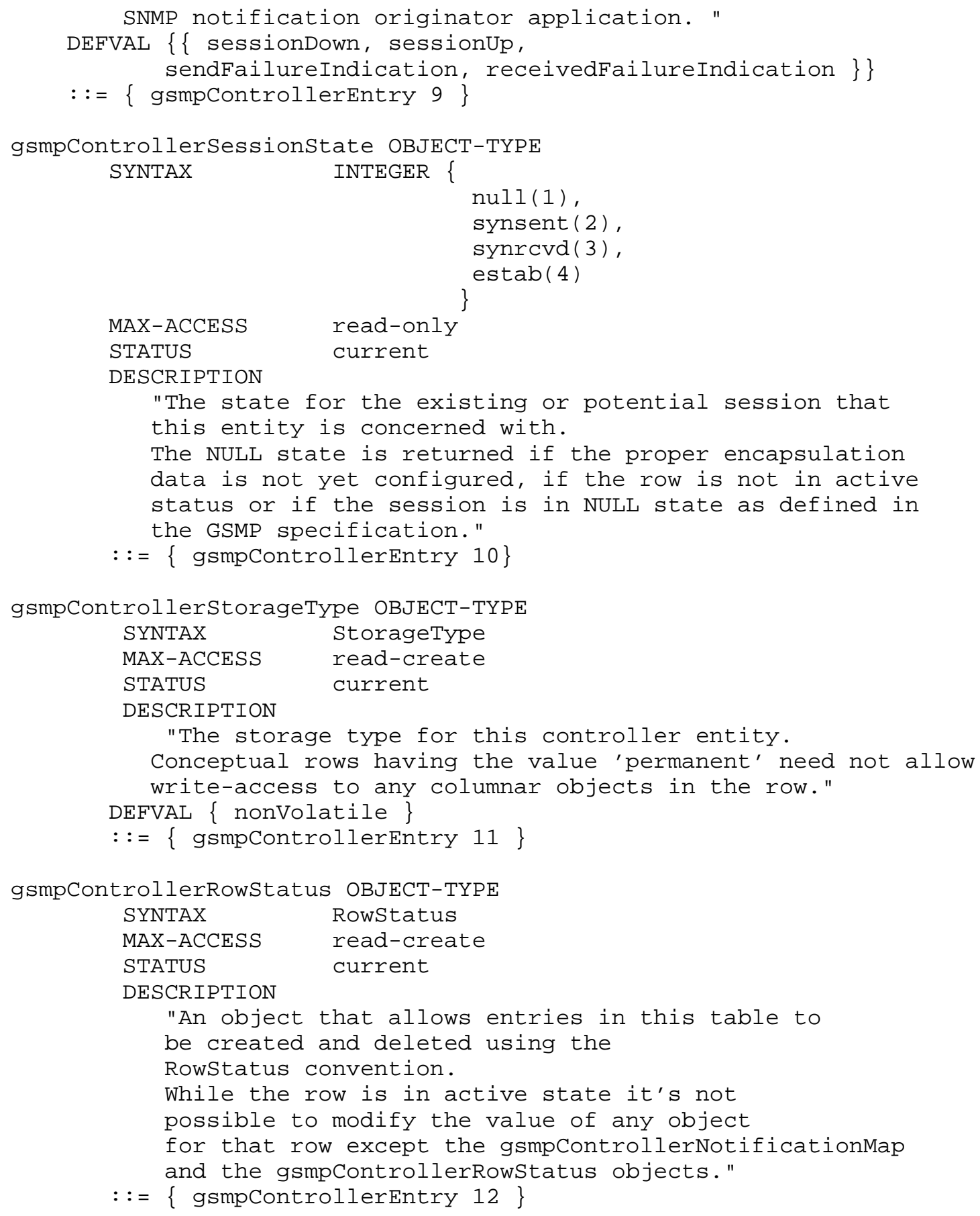




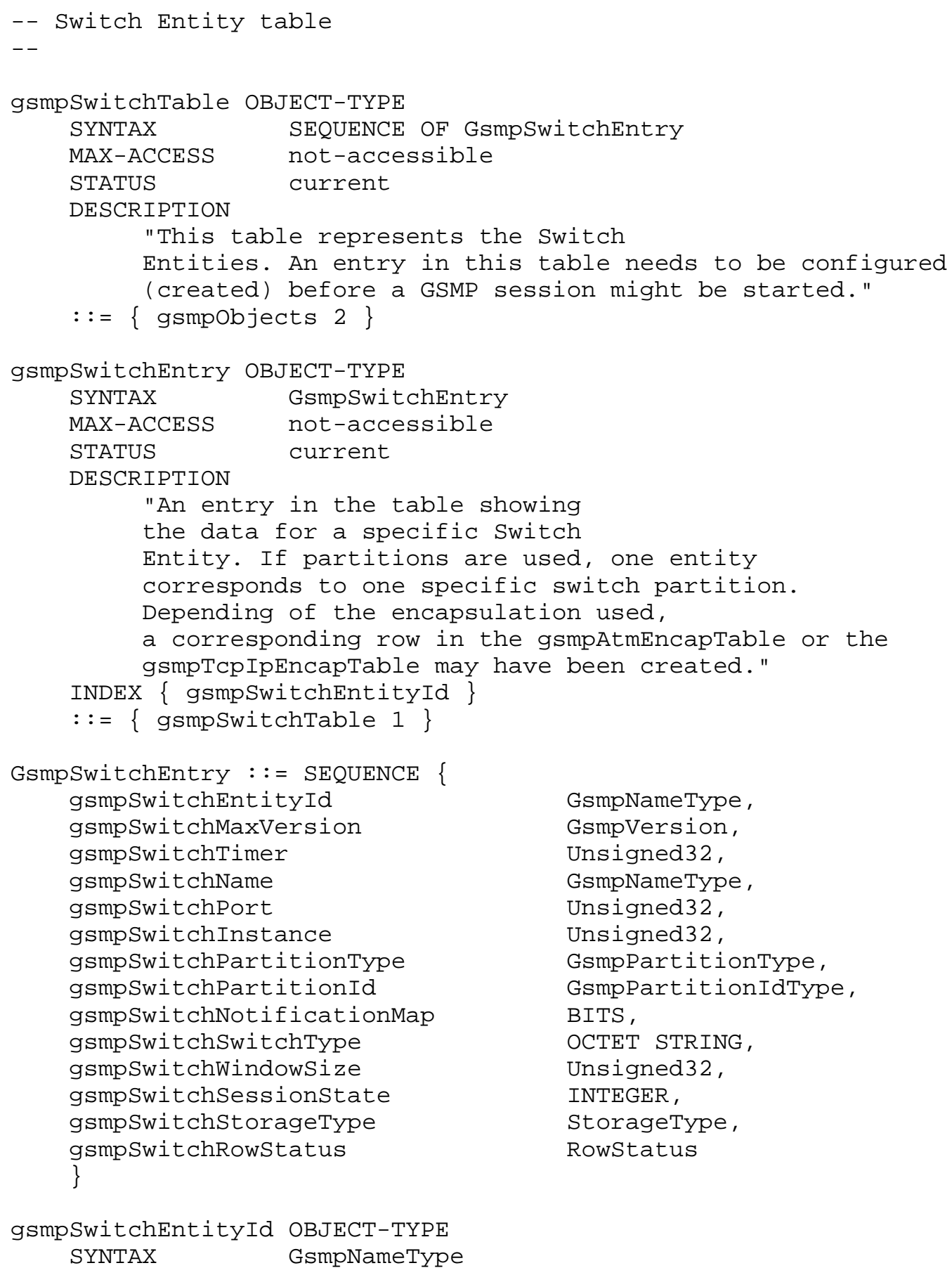




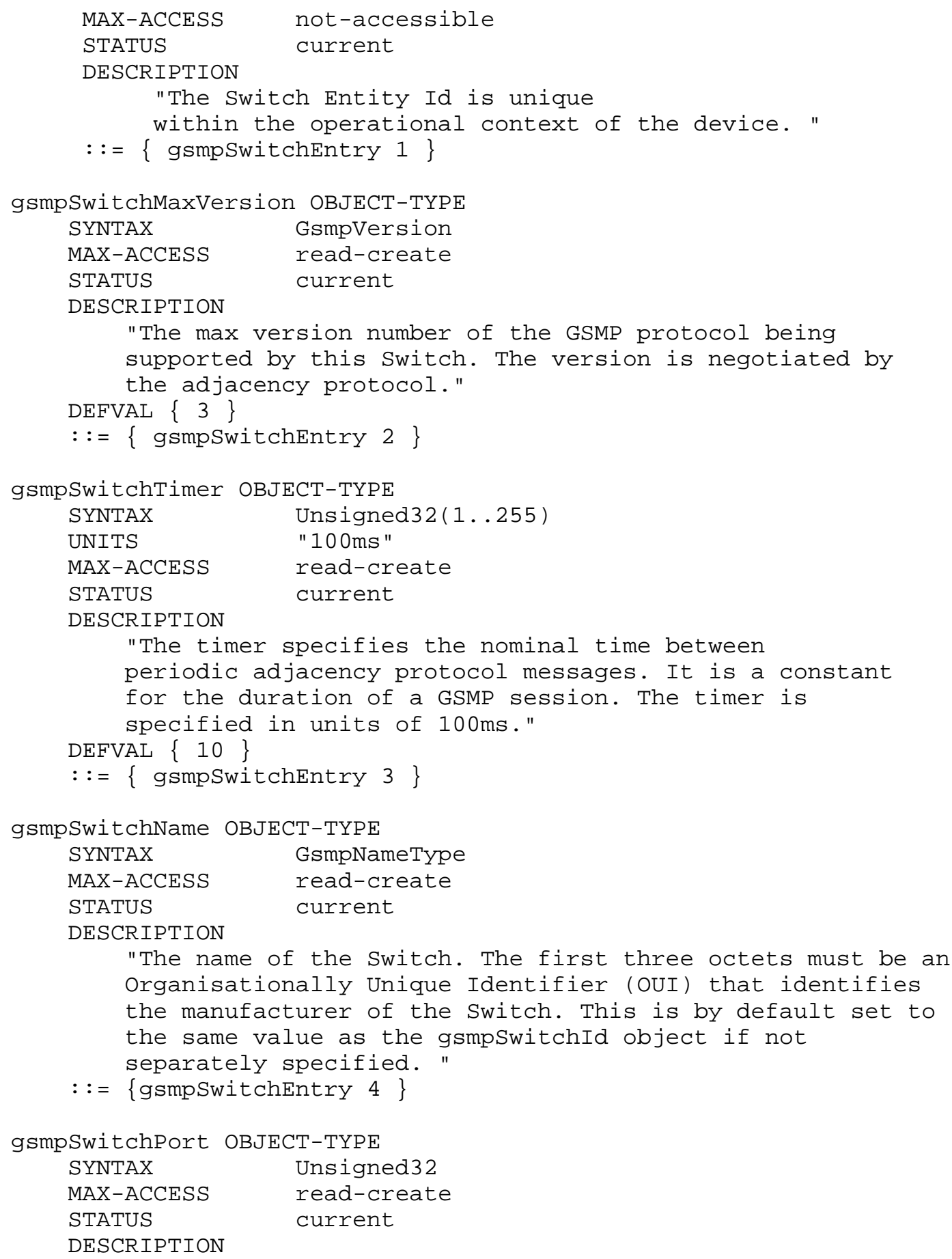




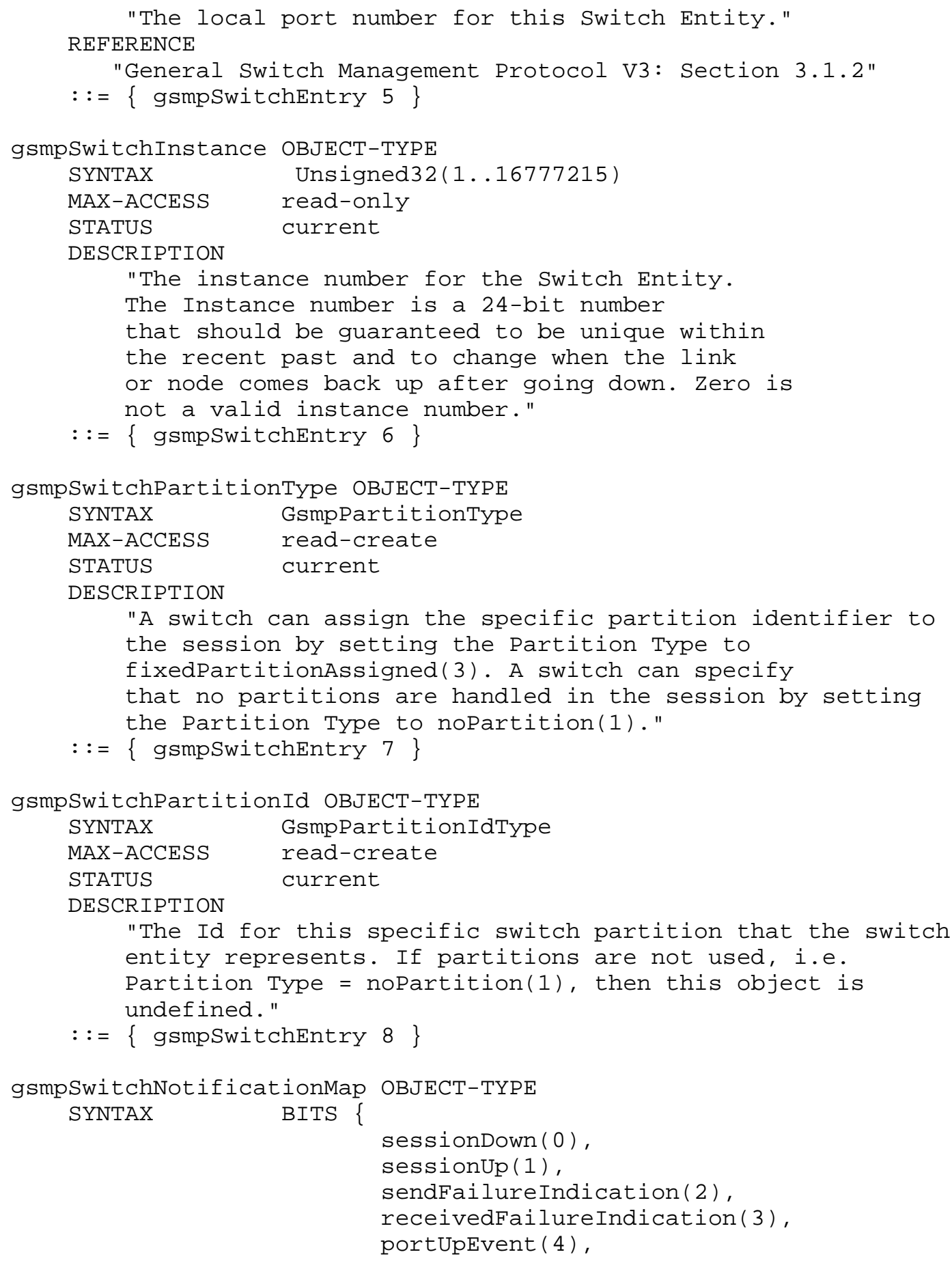




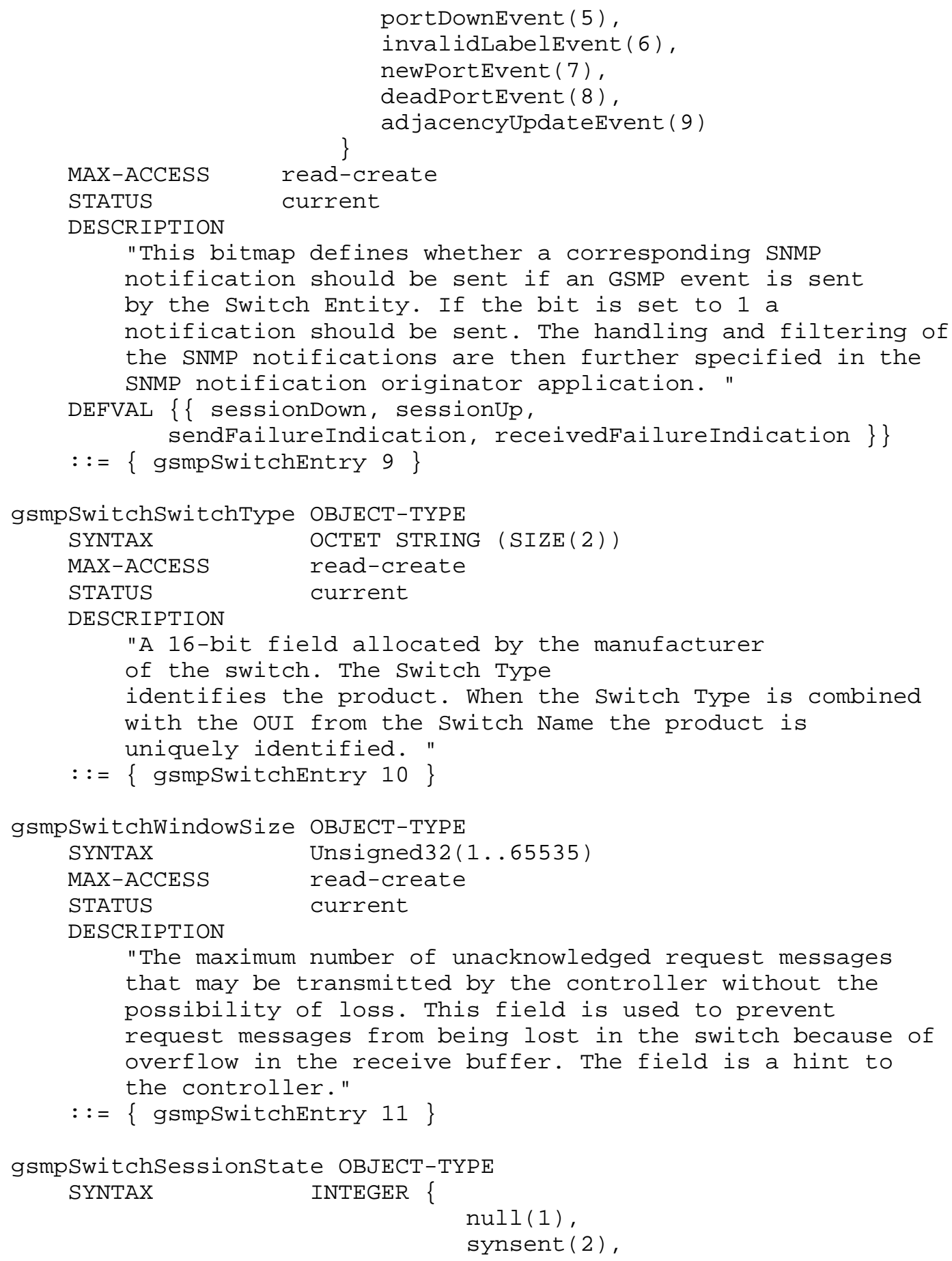




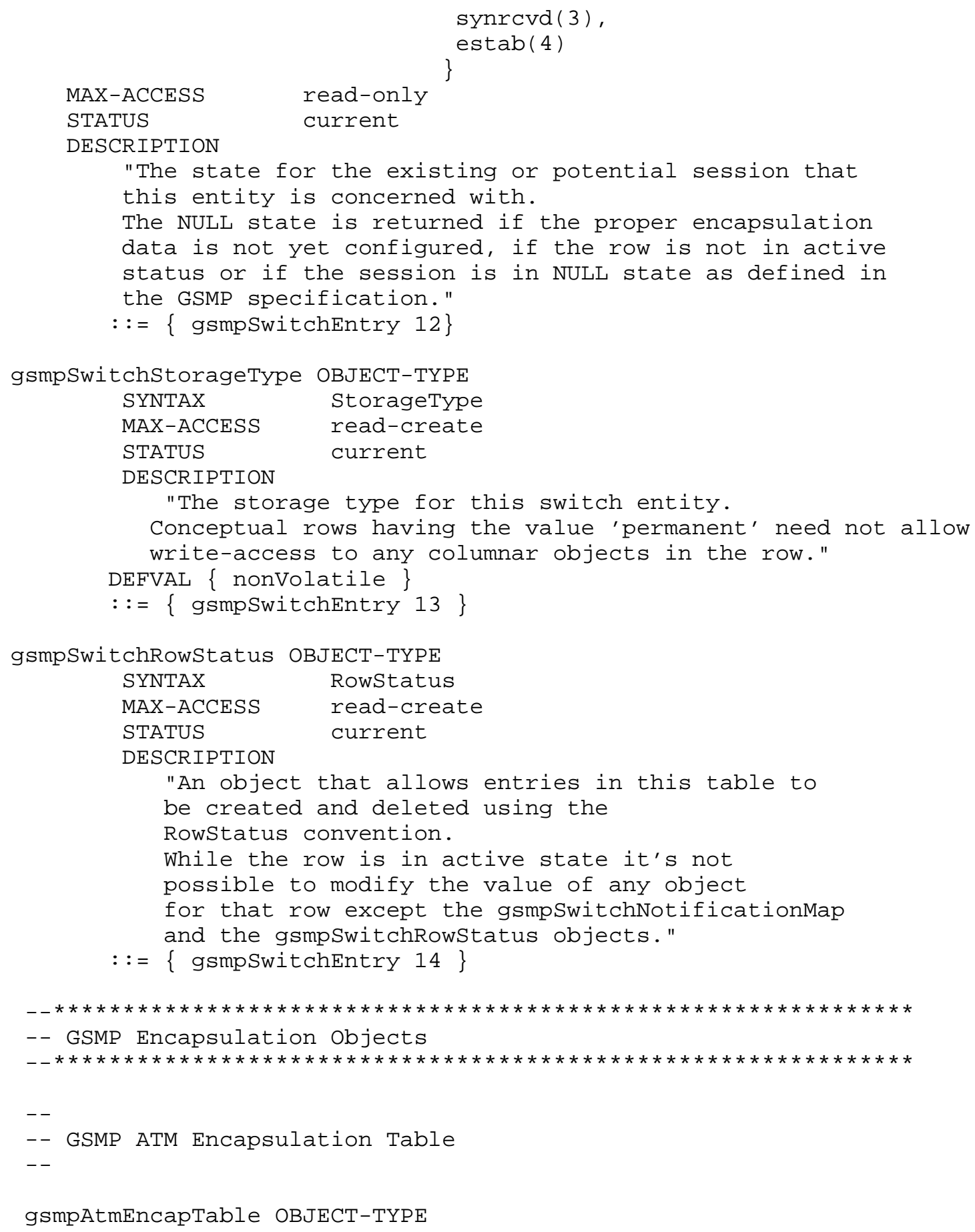




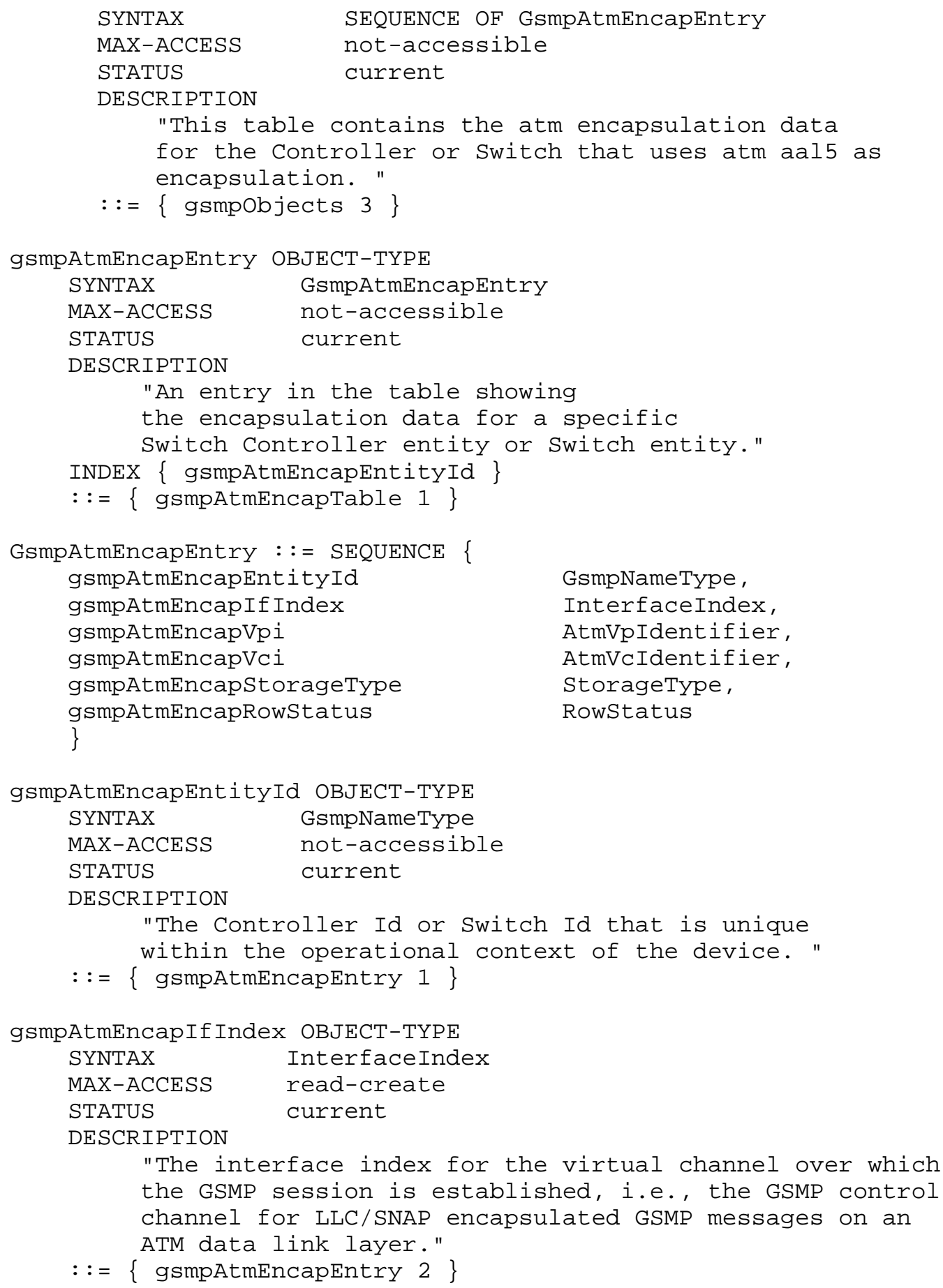




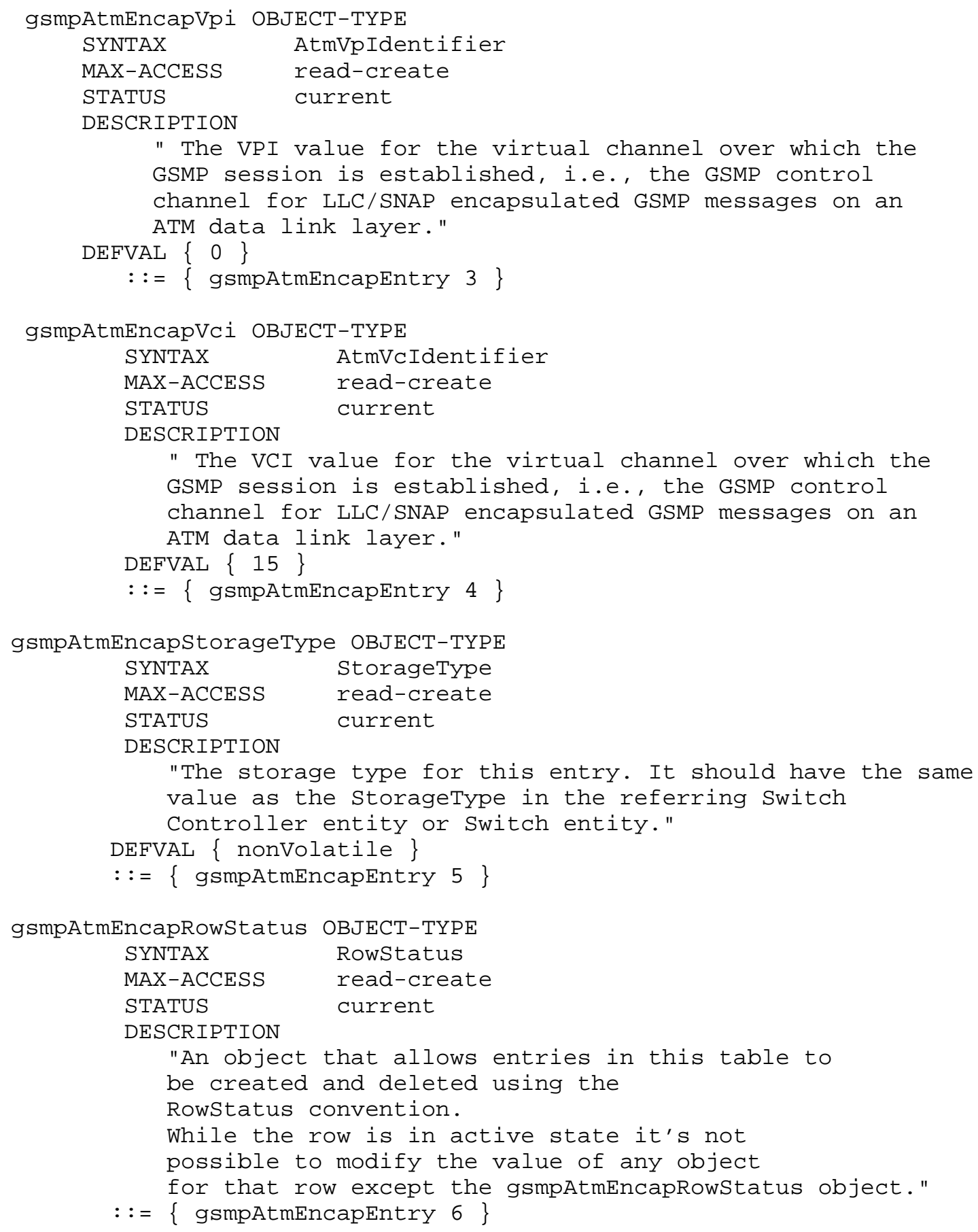




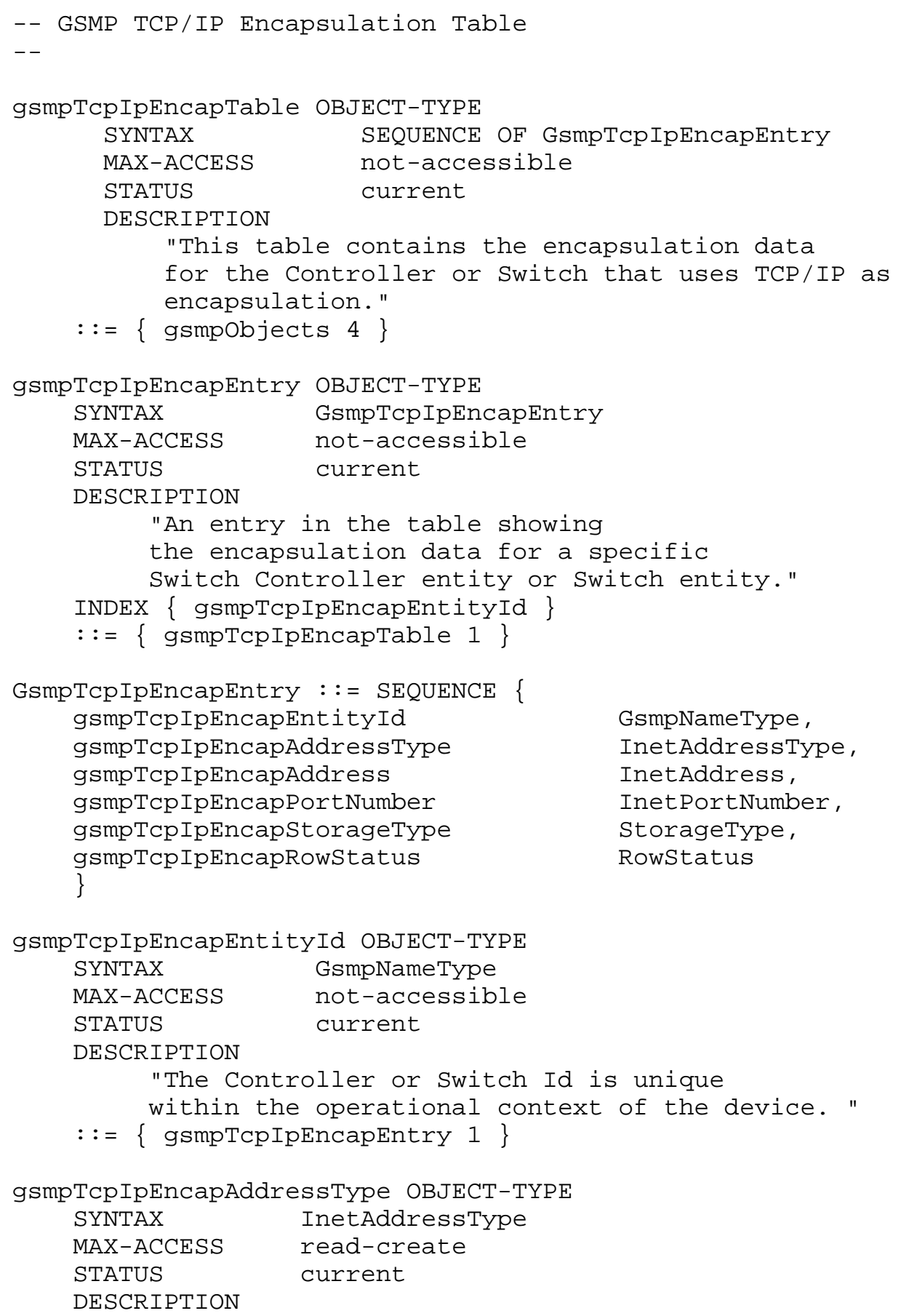




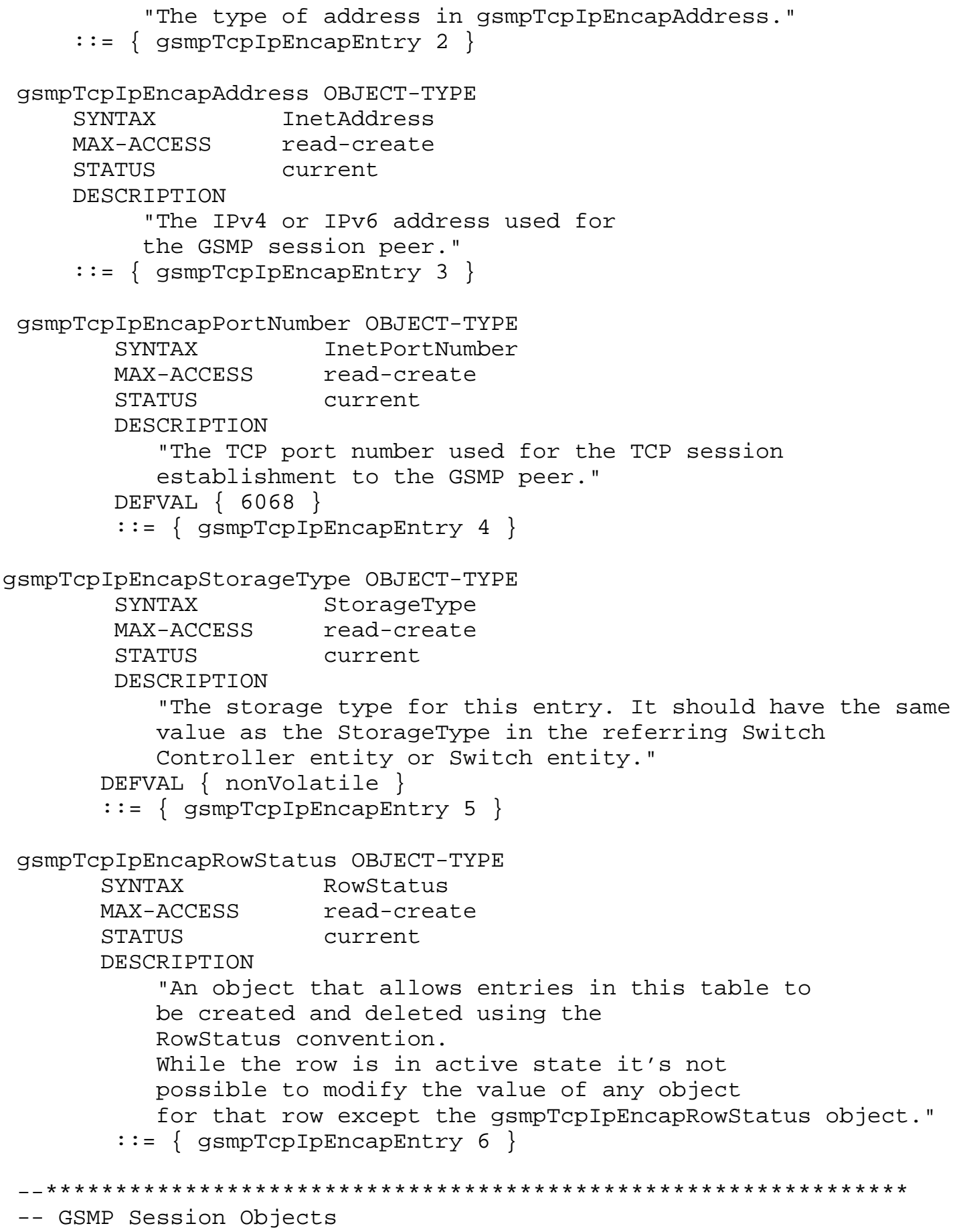




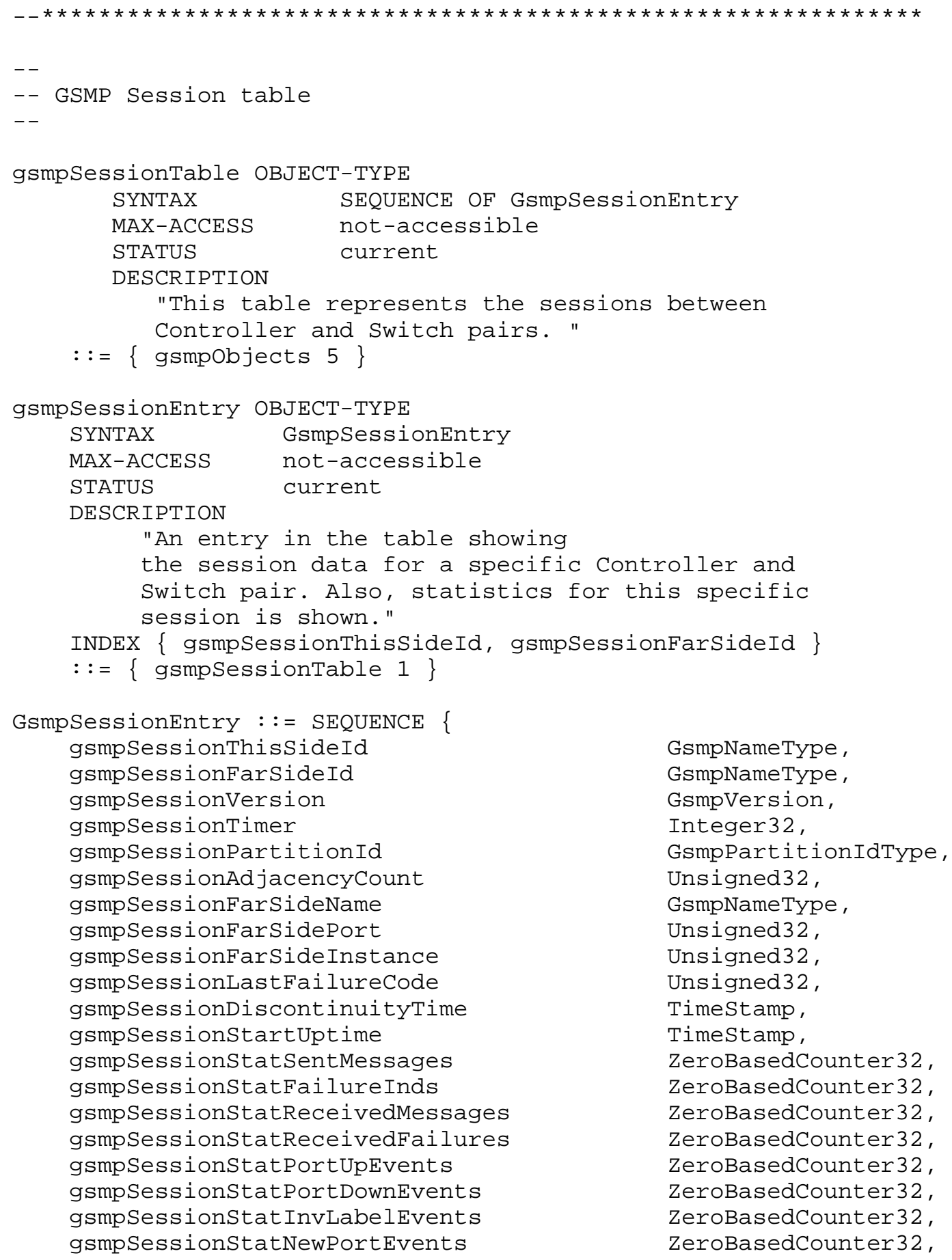

GsmpNameType, GsmpNameType, GsmpVersion, Integer32, GsmpPartitionIdType, Unsigned32, GsmpNameType, Unsigned32, Unsigned32, Unsigned32, Timestamp, Timestamp, ZeroBasedCounter32, ZeroBasedCounter32, ZeroBasedCounter32, ZeroBasedCounter32, ZeroBasedCounter32, ZeroBasedCounter32, ZeroBasedCounter32, ZeroBasedCounter32, 
gsmpSessionstatDeadPortEvents gsmpSessionstatAdjUpdateEvents \}

gsmpSessionThisSideId OBJECT-TYPE

SYNTAX GsmpNameType

MAX-ACCESS not-accessible

STATUS

DESCRIPTION

"This side ID uniquely identifies the entity that this session relates to within the operational context of the device. "

$::=\{$ gsmpsessionEntry 1$\}$
ZeroBasedCounter32, ZeroBasedCounter 32

gsmpSessionFarSideId OBJECT-TYPE

SYNTAX GsmpNameType

MAX-ACCESS not-accessible

STATUS current

DESCRIPTION

"The Far side ID uniquely identifies the entity that this session is established against. "

$::=\{$ gsmpsessionEntry 2$\}$

gsmpSessionVersion OBJECT-TYPE

SYNTAX GsmpVersion

MAX-ACCESS read-only

STATUS current

DESCRIPTION

"The version number of the GSMP protocol being used in

this session. The version is the result of the negotiation by the adjacency protocol."

$::=\{$ gsmpsessionEntry 3$\}$

gsmpSessionTimer OBJECT-TYPE

SYNTAX Integer32

UNITS "100ms"

MAX-ACCESS read-only

STATUS current

DESCRIPTION

"The timer specifies the time remaining until the adjacency timer expires. The object could take negative values since if no valid GSMP messages are

received in any period of time in excess of three times the value of the Timer negotiated by the adjacency protocol loss of synchronisation may be declared. The timer is specified in units of 100ms."

$::=\{$ gsmpSessionEntry 4$\}$ 


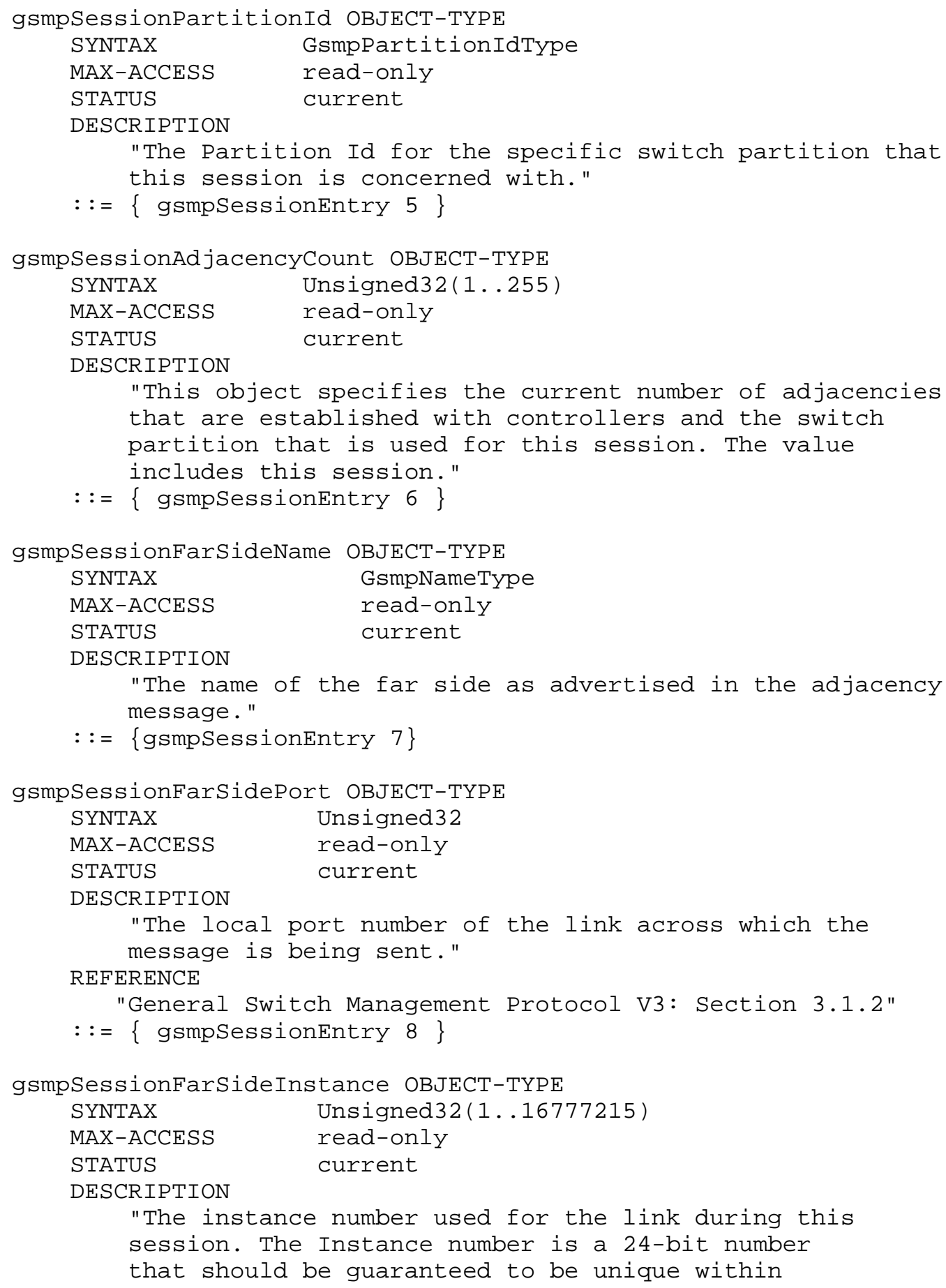




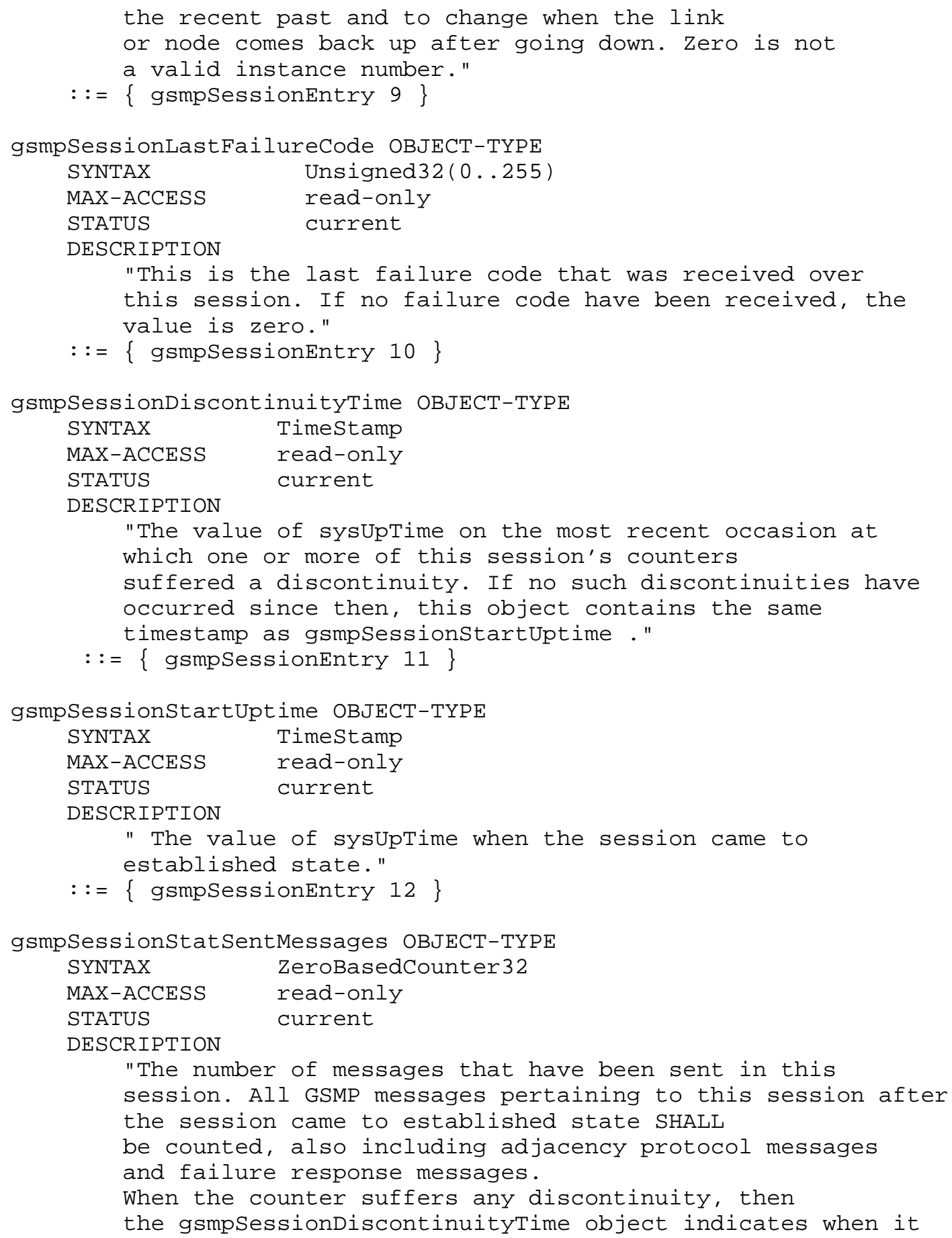




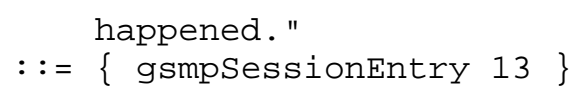




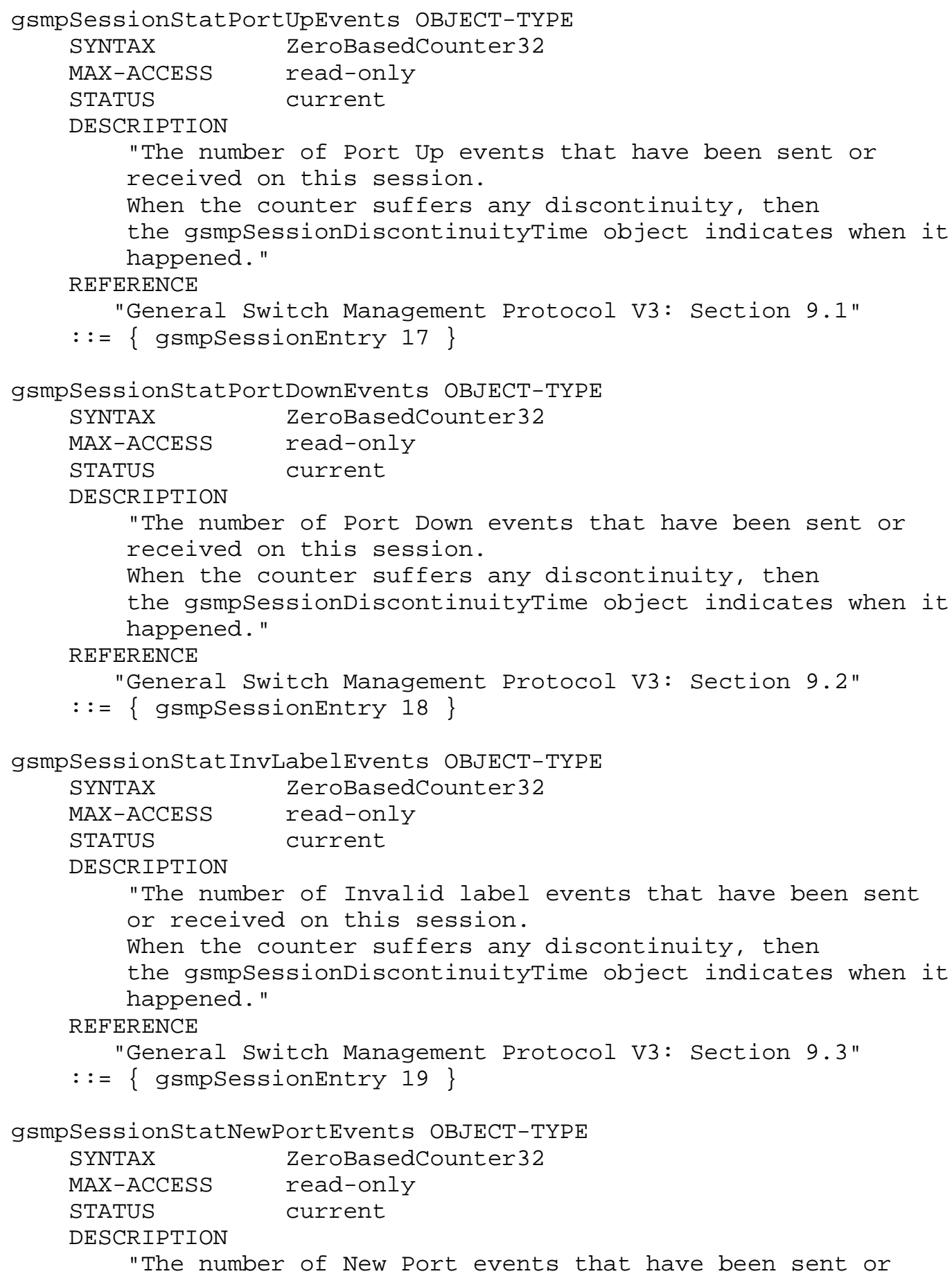

Sjostrand, et. al. Standards Track [Page 30] 


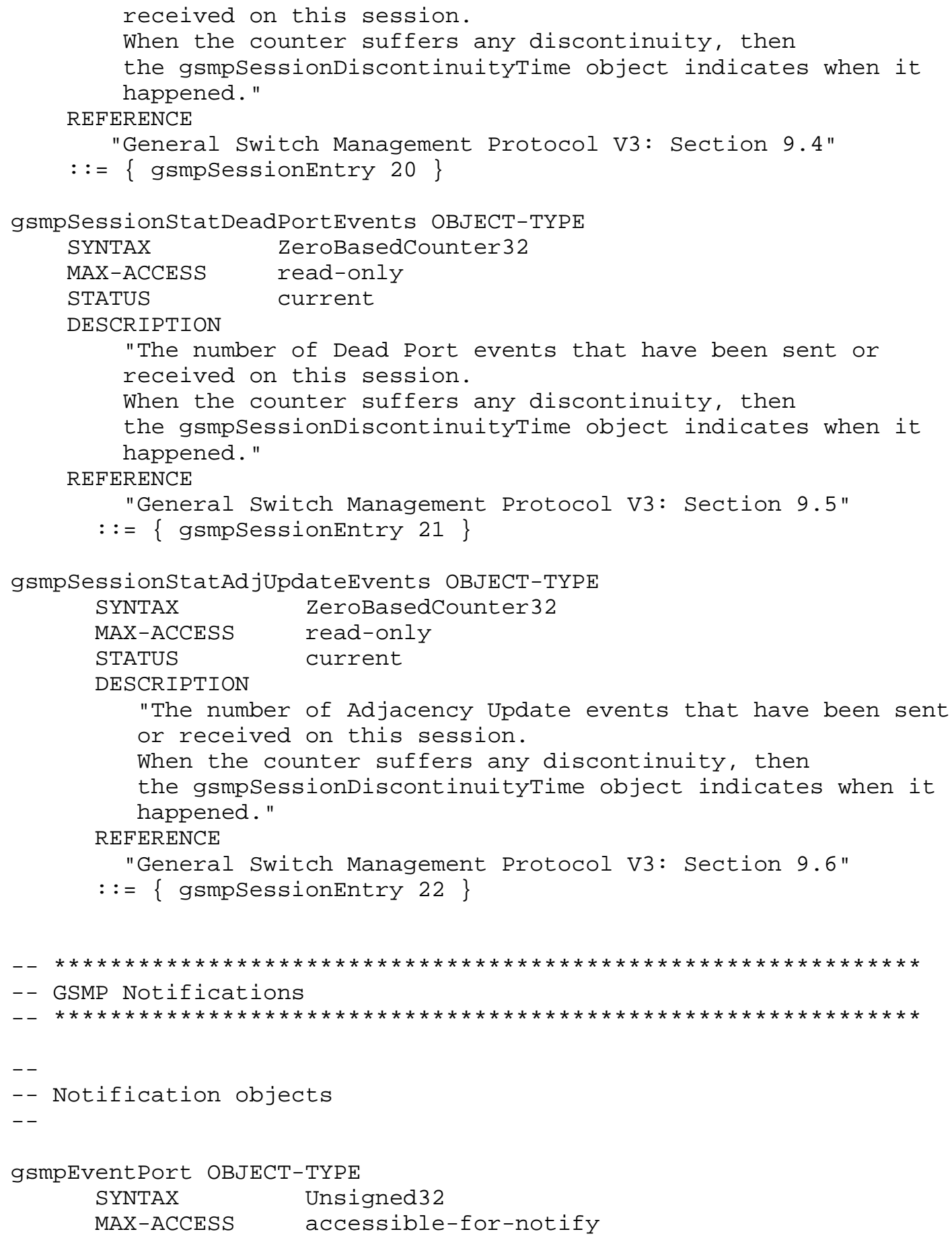




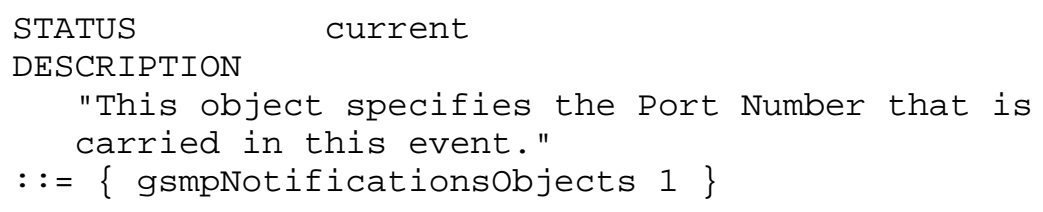

Sjostrand, et. al.

Standards Track

[Page 32] 


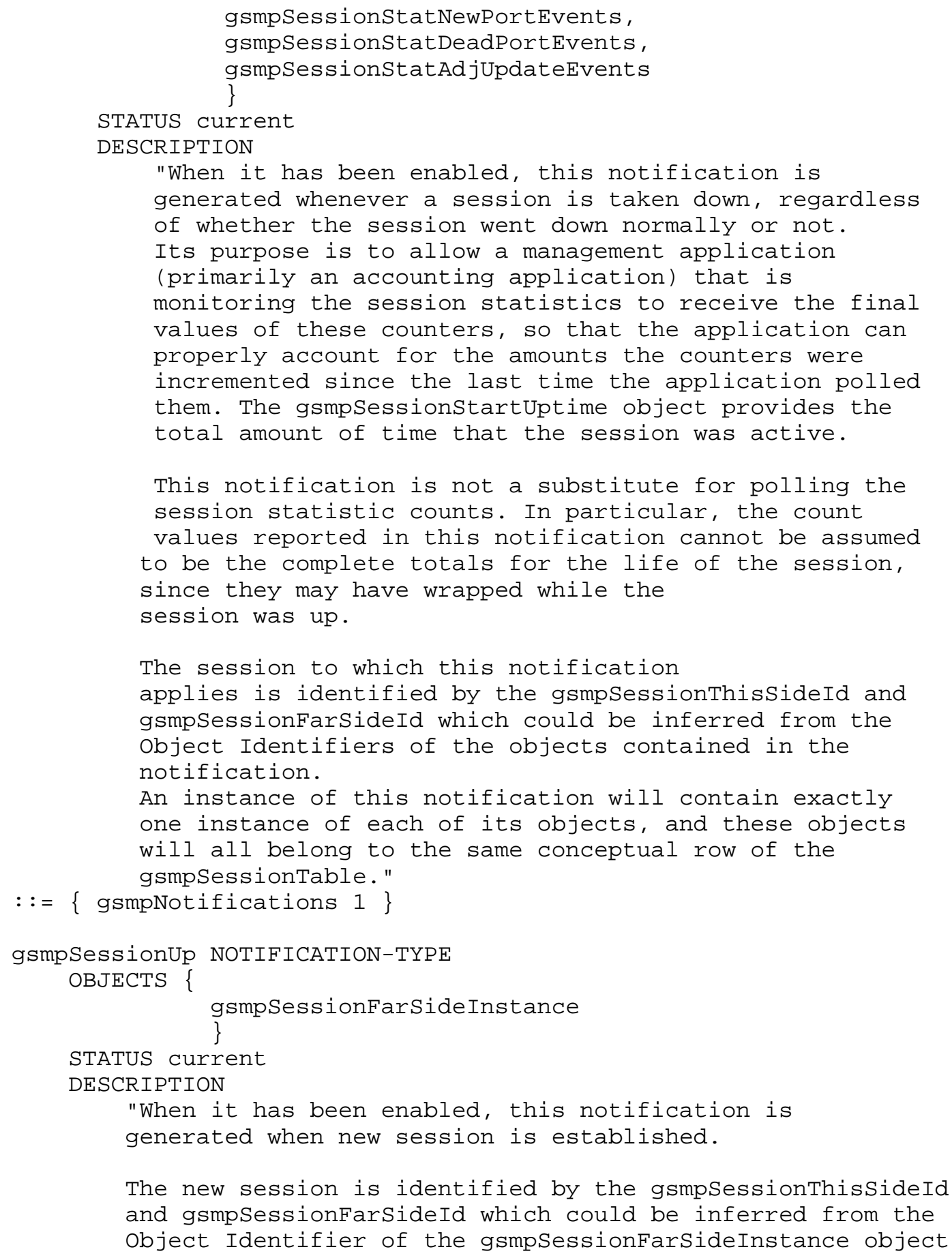


contained in the notification."

$::=\{$ gsmpNotifications 2$\}$

gsmpsentFailureInd NOTIFICATION-TYPE

OBJECTS \{

gsmpSessionLastFailureCode, gsmpSessionstatFailureInds

\}

STATUS current

DESCRIPTION

"When it has been enabled, this notification is

generated when a message with a failure indication was sent.

The notification indicates a change in the value of gsmpSessionstatFailureInds. The gsmpsessionLastFailureCode contains the failure reason.

The session to which this notification applies is identified by the gsmpsessionThissideId and gsmpsessionfarSideId which could be inferred from the object Identifiers of the objects contained in the notification."

$::=\{$ gsmpNotifications 3$\}$

gsmpReceivedFailureInd NOTIFICATION-TYPE OBJECTS \{

gsmpSessionLastFailureCode, gsmpSessionStatReceivedFailures

\}

STATUS current

DESCRIPTION

"When it has been enabled, this notification is

generate when a message with a failure indication is received.

The notification indicates a change in the value of gsmpsessionStatReceivedFailures. The gsmpSessionLastFailureCode contains the failure reason.

The session to which this notification applies is identified by the gsmpsessionThissideId and gsmpsessionfarSideId which could be inferred from the object Identifiers of the objects contained in the notification."

$::=\{$ gsmpNotifications 4$\}$

Sjostrand, et. al.

Standards Track

[Page 34] 


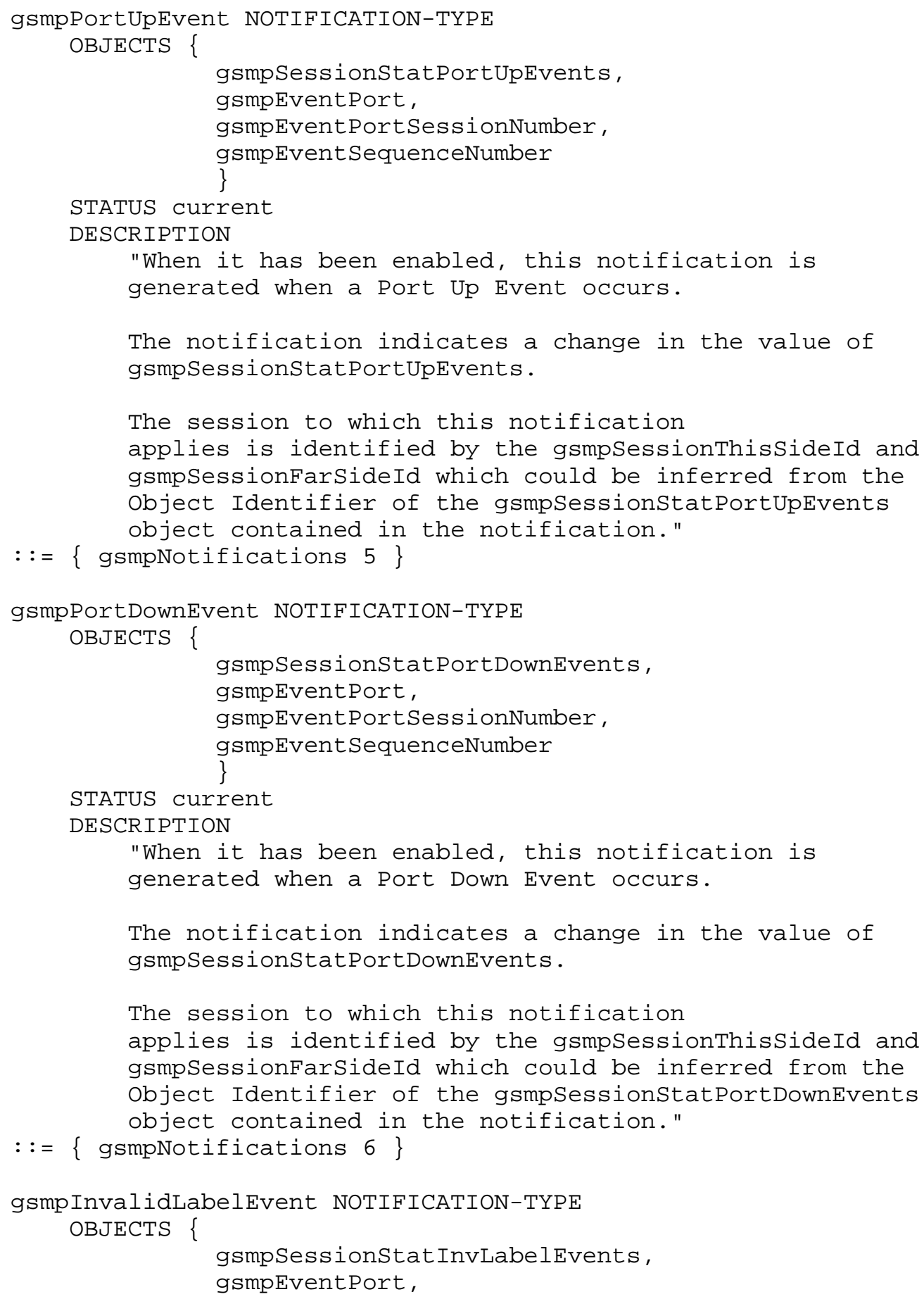




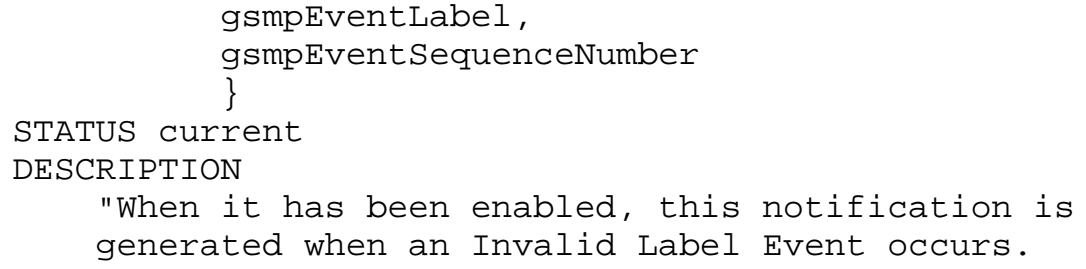

Sjostrand, et. al.

Standards Track

[Page 36] 


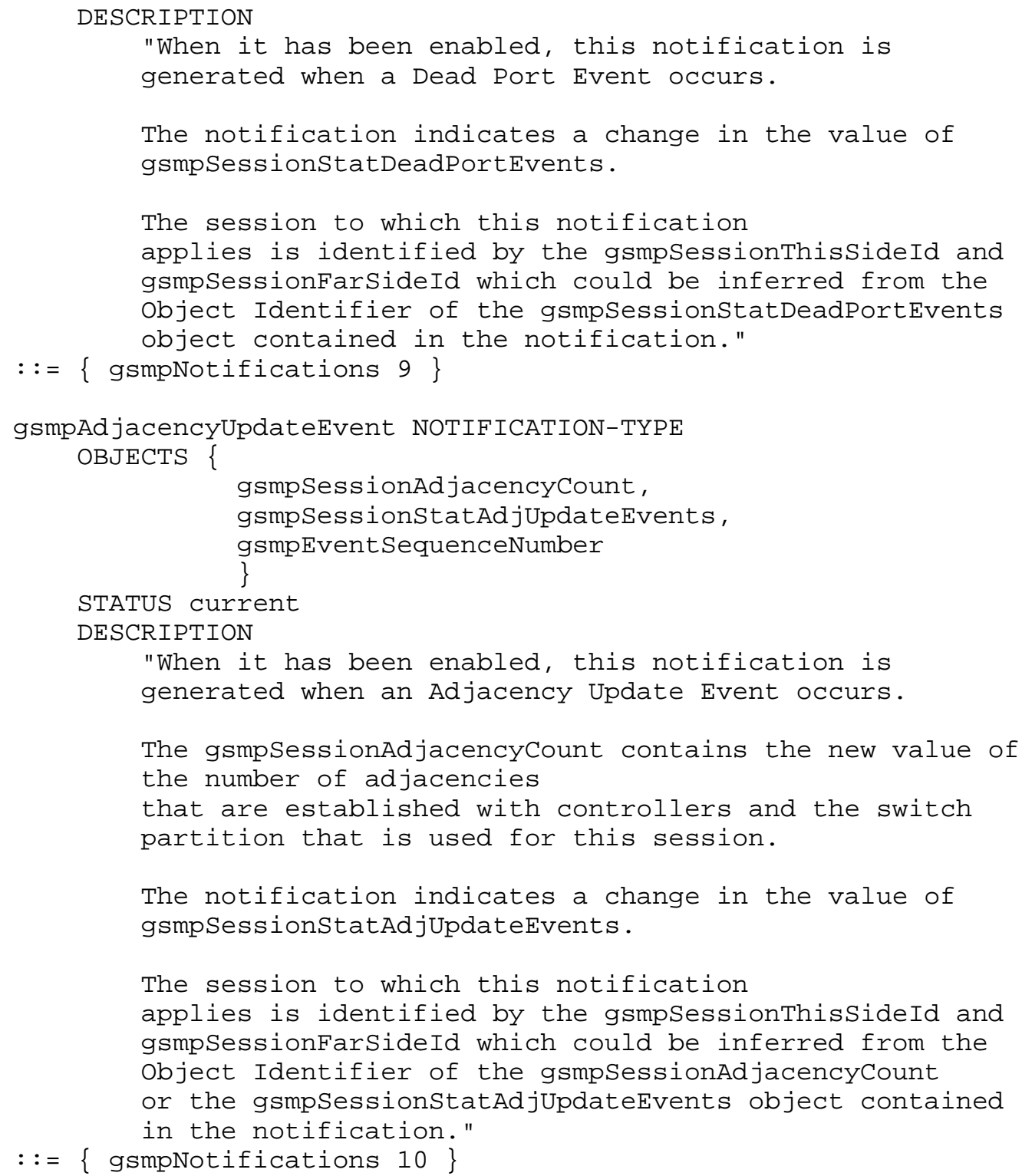




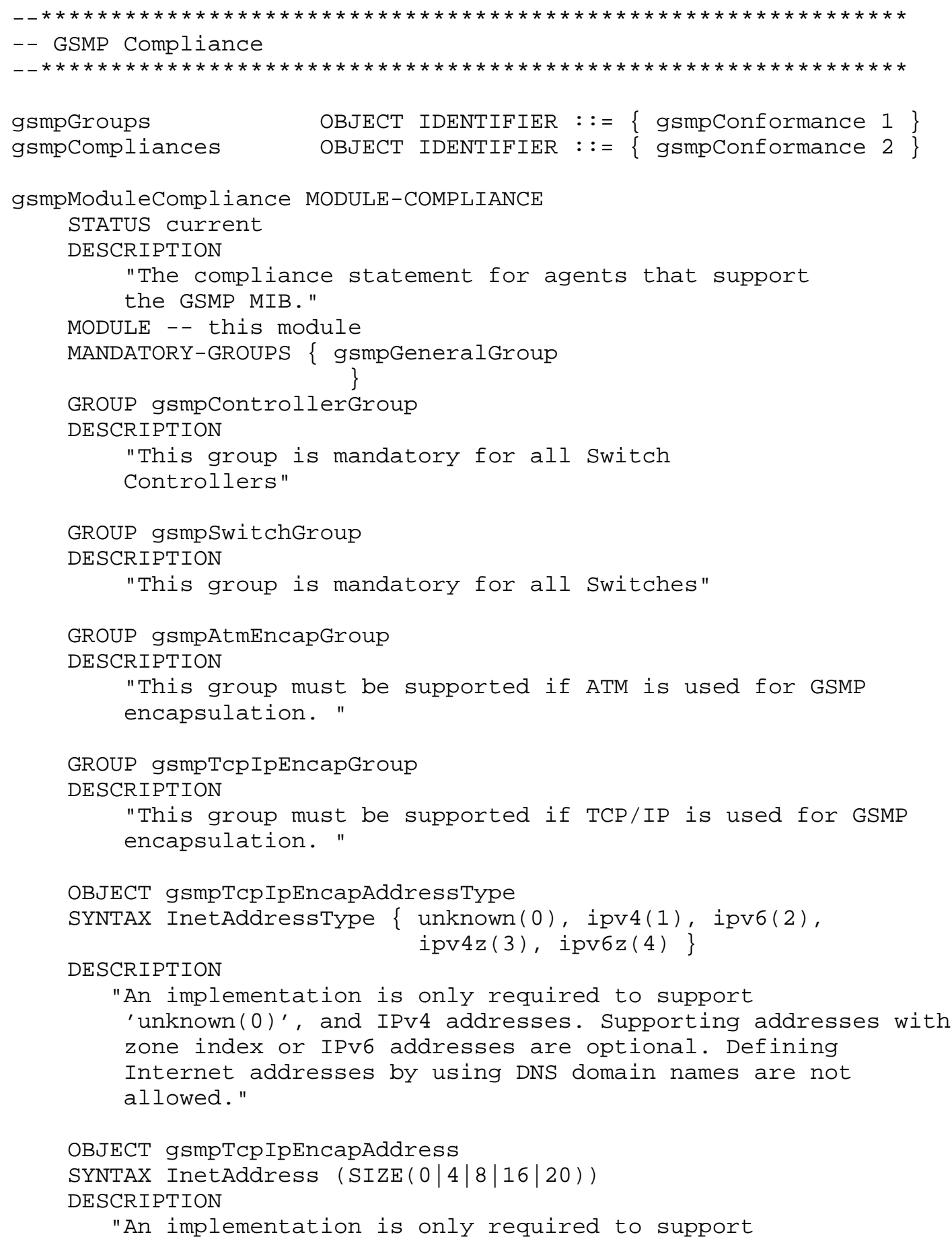


IPv4 addresses. Supporting addresses with zone index or IPv6 addresses are optional."

GROUP gsmpNotificationobjectsGroup

DESCRIPTION

"This group must be supported if notifications

are supported. "

GROUP gsmpNotificationsGroup

DESCRIPTION

"This group must be supported if notifications

are supported. "

$::=\{$ gsmpCompliances 1$\}$

-- units of conformance

gsmpGeneralGroup OBJECT-GROUP

OBJECTS \{

gsmpsessionVersion,

gsmpsessiontimer,

gsmpSessionPartitionId,

gsmpSessionAdjacencyCount, gsmpsessionfarsideName,

gsmpsessionfarsidePort, gsmpSessionfarSideInstance, gsmpSessionLastFailureCode, gsmpsessiondiscontinuityTime, gsmpSessionstartUptime, gsmpSessionstatSentMessages, gsmpSessionStatFailureInds, gsmpSessionstatReceivedMessages, gsmpSessionstatReceivedFailures, gsmpSessionStatPortUpEvents, gsmpsessionstatPortDownEvents, gsmpsessionstat InvLabelEvents, gsmpSessionStatNewPortEvents, gsmpSessionstatDeadPortEvents, gsmpSessionstatAdjUpdateEvents

\}

STATUS current

DESCRIPTION

"Objects that apply to all GSMP implementations." $::=\{$ gsmpGroups 1$\}$

gsmpControllerGroup OBJECT-GROUP

OBJECTS \{

gsmpControllerMaxVersion, 


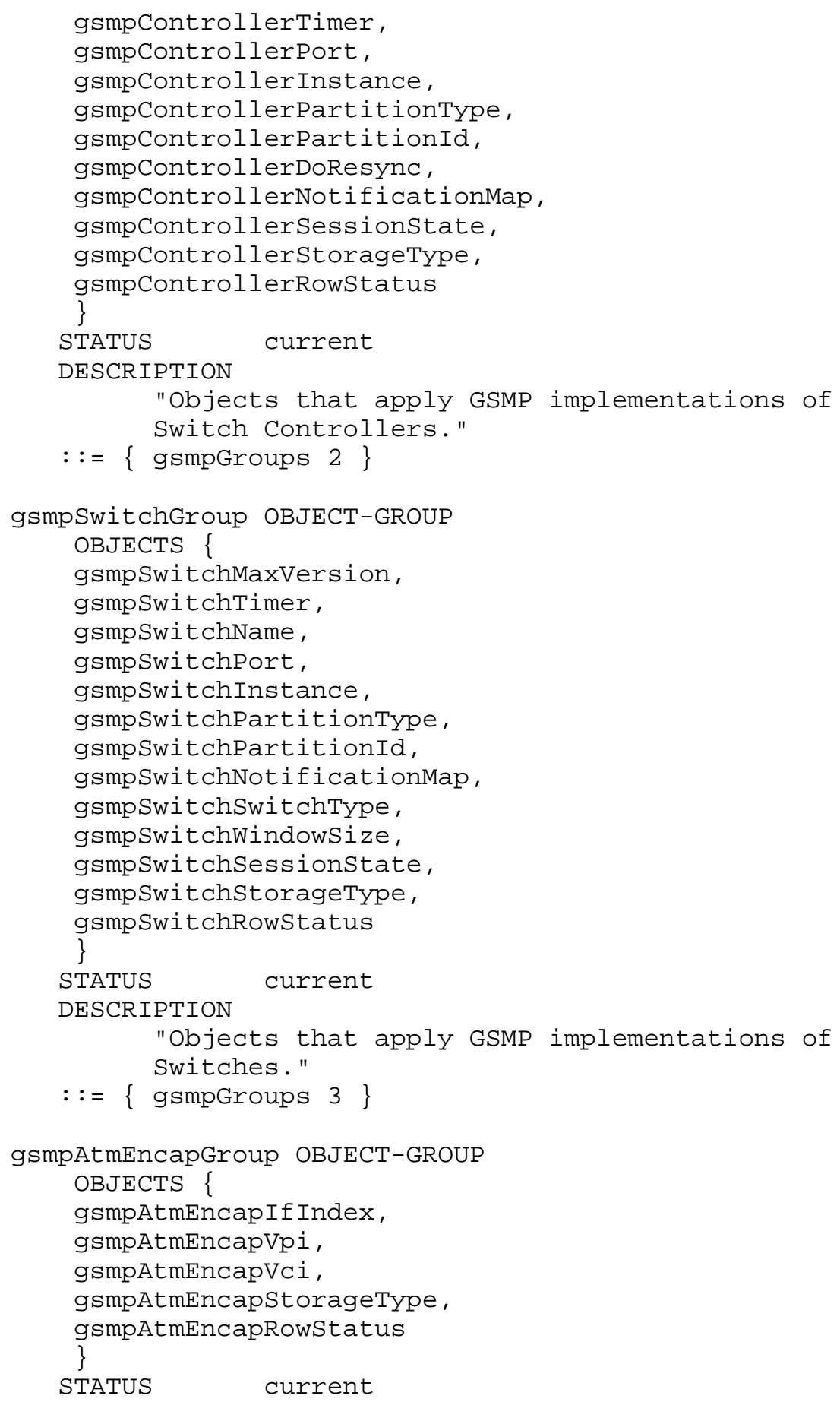




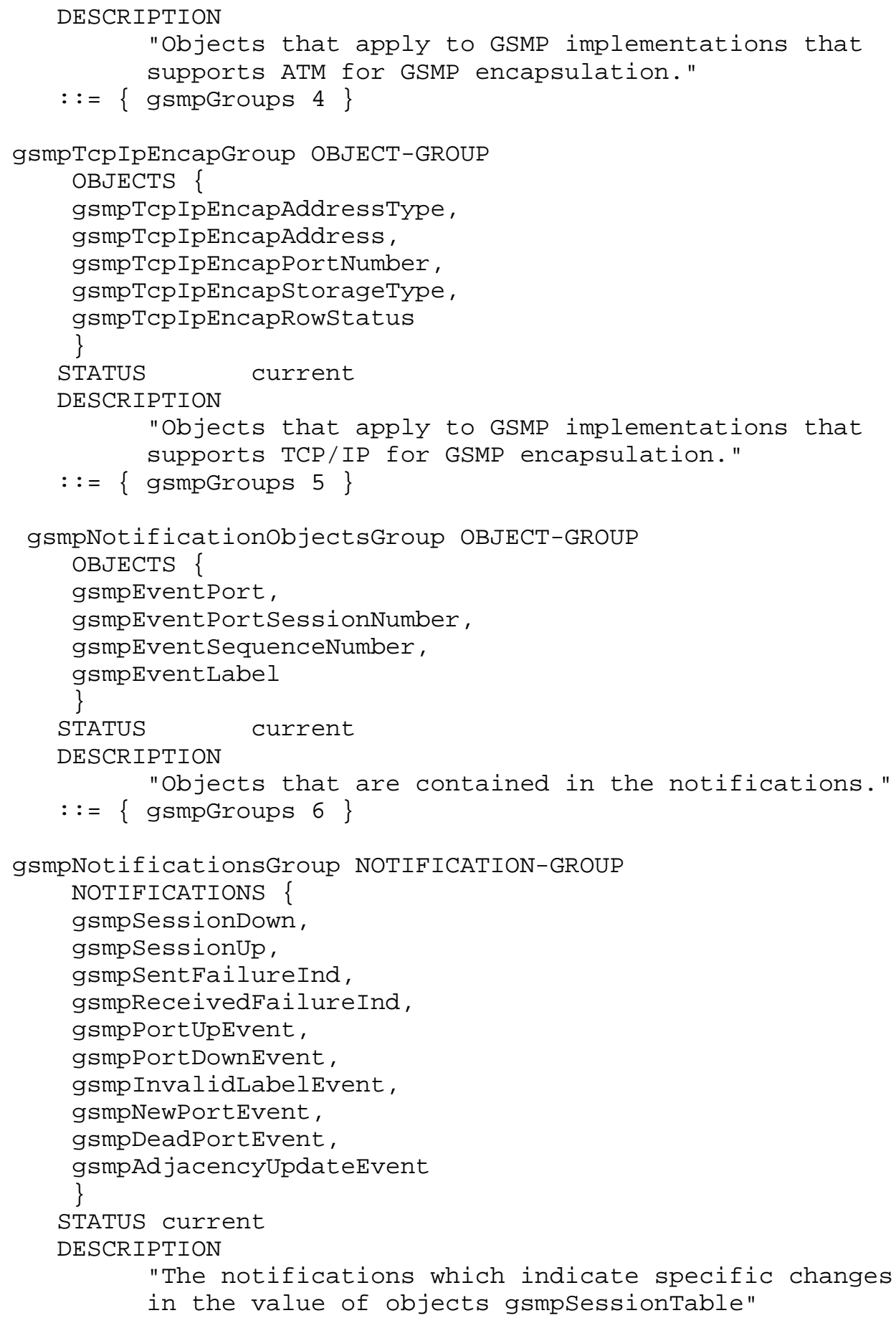


$::=\{$ gsmpGroups 7$\}$

END

5. Acknowledgments

The authors would like to thank Avri Doria and Kenneth Sundell for their contributions to this specification. Also thanks to David Partain, Michael MacFaden and Bert Wijnen who have contributed significantly with their SNMP expertise.

6. References

[RFC1155] Rose, M. and K. McCloghrie, "Structure and Identification of Management Information for TCP/IP-based Internets", STD 16, RFC 1155, May 1990.

[RFC1212] Rose, M. and K. McCloghrie, "Concise MIB Definitions", STD 16, RFC 1212, March 1991.

[RFC1215] Rose, M., "A Convention for Defining Traps for use with the SNMP", RFC 1215, March 1991.

[RFC1157] Case, J., Fedor, M., Schoffstall, M. and J. Davin, "Simple Network Management Protocol", STD 15, RFC 1157, May 1990 .

[RFC1901] Case, J., MCCloghrie, K., Rose, M. and S. Waldbusser, "Introduction to Community-based SNMPv2", RFC 1901, January 1996 .

[RFC1905] Case, J., MCCloghrie, K., Rose, M. and S. Waldbusser, "Protocol Operations for Version 2 of the Simple Network Management Protocol (SNMPv2)", RFC 1905, January 1996.

[RFC1906] Case, J., MCCloghrie, K., Rose, M. and S. Waldbusser, "Transport Mappings for Version 2 of the Simple Network Management Protocol (SNMPv2)", RFC 1906, January 1996.

[RFC1987] Newman, P, Edwards, W., Hinden, R., Hoffman, E., Ching Liaw, F., Lyon, T. and Minshall, G., "Ipsilon's General Switch Management Protocol Specification," Version 1.1, RFC 1987, August 1996.

[RFC2021] Waldbusser, S., "Remote Network Monitoring Management Information Base Version 2 using SMIv2", RFC 2021, January 1997 . 
[RFC2026] Bradner, S., "The Internet Standards Process - Revision 3", BCP 9, RFC 2026, October 1996.

[RFC2119] Bradner, S., "Key words for use in RFCs to Indicate Requirement Levels", BCP 14, RFC 2119, March 1997.

[RFC2397] Newman, P, Edwards, W., Hinden, R., Hoffman, E., Ching Liaw, F., Lyon, T. and Minshall, G., "Ipsilon's General Switch Management Protocol Specification," Version 2.0, RFC 2397, March 1998.

[RFC2434] Narten, T. and H. Alvestrand, "Guidelines for Writing an IANA Considerations Section in RFCs.", BCP 26, RFC 2434, October 1998 .

[RFC2514] Noto, M., E. Spiegel, K. Tesink, "Definition of Textual Conventions and OBJECT-IDENTITIES for ATM Management", RFC 2514, February 1999.

[RFC2570] Case, J., Mundy, R., Partain, D. and B. Stewart, "Introduction to Version 3 of the Internet-standard Network Management Framework", RFC 2570, April 1999.

[RFC2571] Harrington, D., Presuhn, R. and B. Wijnen, "An Architecture for Describing SNMP Management Frameworks", RFC 2571, April 1999.

[RFC2572] Case, J., Harrington D., Presuhn R. and B. Wijnen, "Message Processing and Dispatching for the Simple Network Management Protocol (SNMP)", RFC 2572, April 1999 .

[RFC2573] Levi, D., Meyer, P. and B. Stewart, "SNMP Applications", RFC 2573, April 1999.

[RFC2574] Blumenthal, U. and B. Wijnen, "User-based Security Model (USM) for version 3 of the Simple Network Management Protocol (SNMPv3)", RFC 2574, April 1999.

[RFC2575] Wijnen, B., Presuhn, R. and K. McCloghrie, "View-based Access Control Model (VACM) for the Simple Network Management Protocol (SNMP)", RFC 2575, April 1999.

[RFC2578] McCloghrie, K., Perkins, D., Schoenwaelder, J., Case, J., Rose, M. and S. Waldbusser, "Structure of Management Information Version 2 (SMIV2)", STD 58, RFC 2578, April 1999. 
[RFC2579] McCloghrie, K., Perkins, D., Schoenwaelder, J., Case, J., Rose, M. and S. Waldbusser, "Textual Conventions for SMIv2", STD 58, RFC 2579, April 1999.

[RFC2580] McCloghrie, K., Perkins, D., Schoenwaelder, J., Case, J., Rose, M. and S. Waldbusser, "Conformance Statements for SMIV2", STD 58, RFC 2580, April 1999.

[RFC2863] MCCloghrie, K. and F. Kastenholz, "The Interfaces Group MIB" RFC 2863, June 2000 .

[RFC3291] Daniele, M., Haberman, B., Routhier, S. and J., Schoenwaelder "Textual Conventions for Internet Network Addresses", RFC 3291, May 2002.

[RFC3292] Doria, A., Hellstrand, F., Sundell, K. and T. Worster, "General Switch Management Protocol V3", RFC 3292, June 2002 .

[RFC3293] Worster, T., Doria, A. and J. Buerkle, "General Switch Management Protocol (GSMP) Packet Encapsulations for Asynchronous Transfer Mode (ATM), Ethernet and Transmission Control Protocol (TCP)", RFC 3293, June 2002 .

7. Intellectual Property Rights

The IETF takes no position regarding the validity or scope of any intellectual property or other rights that might be claimed to pertain to the implementation or use of the technology described in this document or the extent to which any license under such rights might or might not be available; neither does it represent that it has made any effort to identify any such rights. Information on the IETF's procedures with respect to rights in standards-track and standards-related documentation can be found in BCP-11. Copies of claims of rights made available for publication and any assurances of licenses to be made available, or the result of an attempt made to obtain a general license or permission for the use of such proprietary rights by implementors or users of this specification can be obtained from the IETF secretariat.

The IETF invites any interested party to bring to its attention any copyrights, patents or patent applications, or other proprietary rights which may cover technology that may be required to practice this standard. Please address the information to the IETF Executive Director. 


\section{Security Considerations}

Assuming that secure network management (such as SNMP v3) is implemented, the objects represented in this MIB do not pose a threat to the security of the network.

There are a number of management objects defined in this MIB that have a MAX-ACCESS clause of read-write and/or read-create. Such objects may be considered sensitive or vulnerable in some network environments. The support for SET operations in a non-secure environment without proper protection can have a negative effect on network operations.

There are a number of managed objects in this MIB that may contain sensitive information. They are contained in the gsmpcontrollerTable and gsmpswitchTable. It is thus important to control even GET access to these objects and possibly to even encrypt the values of these object when sending them over the network via SNMP. Not all versions of SNMP provide features for such a secure environment.

SNMPv1 by itself is not a secure environment. Even if the network itself is secure (for example by using IPSec), even then, there is no control as to who on the secure network is allowed to access and $\mathrm{GET} / \mathrm{SET}$ (read/change/create/delete) the objects in this MIB.

It is recommended that the implementers consider the security features as provided by the SNMPv3 framework. Specifically, the use of the User-based Security Model RFC 2574 [RFC2574] and the Viewbased Access Control Model RFC 2575 [RFC2575] is recommended.

It is then a customer/user responsibility to ensure that the SNMP entity giving access to an instance of this MIB, is properly configured to give access to the objects, only to those principals (users) that have legitimate rights to indeed GET or SET (change/create/delete) them. 
9. Authors' Addresses

Hans Sjostrand

ipUnplugged

P.O. Box 10160

S-121 28 Stockholm, Sweden

Phone: +4687255930

EMail: hanseipunplugged.com

Joachim Buerkle

Nortel Networks Germany GmbH \& Co. KG

Hahnstrasse 37-39

D-60528 Frankfurt am Main, Germany

Phone: +49696697 3281

EMail: joachim.buerkleanortelnetworks.com

Balaji Srinivasan

CPlane Inc.

897 Kifer Road

Sunnyvale, CA 94086

Phone: +1 4087894099

EMail: balaji@cplane.com 
10. Full Copyright statement

Copyright (C) The Internet Society (2002). All Rights Reserved.

This document and translations of it may be copied and furnished to others, and derivative works that comment on or otherwise explain it or assist in its implementation may be prepared, copied, published and distributed, in whole or in part, without restriction of any kind, provided that the above copyright notice and this paragraph are included on all such copies and derivative works. However, this document itself may not be modified in any way, such as by removing the copyright notice or references to the Internet society or other Internet organizations, except as needed for the purpose of developing Internet standards in which case the procedures for copyrights defined in the Internet Standards process must be followed, or as required to translate it into languages other than English.

The limited permissions granted above are perpetual and will not be revoked by the Internet society or its successors or assigns.

This document and the information contained herein is provided on an "AS IS" basis and THE INTERNET SOCIETY AND THE INTERNET ENGINEERING TASK FORCE DISCLAIMS ALL WARRANTIES, EXPRESS OR IMPLIED, INCLUDING BUT NOT LIMITED TO ANY WARRANTY THAT THE USE OF THE INFORMATION HEREIN WILL NOT INFRINGE ANY RIGHTS OR ANY IMPLIED WARRANTIES OF MERCHANTABILITY OR FITNESS FOR A PARTICULAR PURPOSE.

Acknowledgement

Funding for the RFC Editor function is currently provided by the Internet society. 